\title{
Der Fall Rumsfeld - ein Begräbnis Dritter Klasse für das Völkerstrafgesetzbuch? ${ }^{1}$
}

\section{A. Einleitung}

Zweiter Aufschlag - so lässt sich die aktuelle Situation des Versuchs beschreiben, hochrangige zivile und militärische Verantwortliche der Vereinigten Staaten von Amerika, namentlich den ehemaligen Verteidigungsminister Donald Rumsfeld, in Deutschland strafrechtlich für Menschenrechtsverletzungen im Zuge des »Kriegs gegen den Terror « zu belangen. Im Jahr 2004 wurde nach Bekanntwerden der Folterbilder aus dem Gefängnis Abu Ghraib im Irak eine Strafanzeige gegen ihn und eine ganze Reihe weiterer militärischer und ziviler Verantwortungsträger beim Generalbundesanwalt unter Berufung auf das 2002 erlassene VStGB eingereicht ${ }^{2}$. Die Anzeigeerstatter waren Opfer von Abu Ghraib, die von der US-Menschenrechtsorganisation Center for Constitutional Rights unterstützt wurden. Diese NGO hatte sich zu einer Strafanzeige in Deutschland entschlossen, als deutlich wurde, dass die strafrechtliche Reaktion der US-Behörden sich ausschließlich auf die unmittelbar handelnden Soldaten beschränken und die Taten als Exzesse einzelner dargestellt würden. Nachdem die Generalbundesanwaltschaft die Aufnahme von Ermittlungen wegen dieser Anzeige zwei Tage vor Eröffnung der Münchener Sicherheitskonferenz im Februar 2005, zu der Rumsfeld als amtierender Verteidigungsminister der USA erwartet wurde, abgelehnt hatte ${ }^{3}$, wurde diese Ablehnung im September 2005 vom OLG Stuttgart bestätigt $^{4}$. Diese Entscheidung, in der sich ein deutsches Gericht das erste Mal mit einem Verfahren nach dem VStGB beschäftigte, wurde zu Recht sehr kritisch kommentiert ${ }^{5}$.

Die Anzeigeerstatter entschlossen sich, statt zu einem weiteren Gang durch die Instanzen, ihre Anzeige, angereichert um zusätzliche Fakten und weitere einbezogene Taten und Ver-

1 Herrn stud iur. Bijan Tavakoli sei Dank ausgesprochen für die Unterstützung vor allem bezüglich originär völkerrechtlicher Fragen.

2 Der Text dieser Anzeige ist in zwei Teilen vollständig zugänglich unter www.ccr-ny.org/ v2/legal/september_11th/docs/complaint_in\%20German1_dienstag1.pdf und <www.ccrny.org/v2/legal/september_11th/docs/complaint_In-German2_dienstag2.pdf $>$ sowie in einer bearbeiteten Fassung unter <www.rav.de/download/Strafanzeige_5.pdf $>$ (diese Links wurden, wie alle weiteren genannten, zuletzt am 10.11.2007 überprüft). Letztere wird in der Folge den Zitaten zugrunde gelegt. Siehe hierzu auch Basak HuV-I 2005, S. 85 ff.; FischerLescano KJ 2005, S. 72 ff.; Hessbrügge ASIL Insights December 2004 (<www.asil.org/ insights/2004/12/insight041213.htm>); Kurth ZIS 2006, S. 81 ff. (zugänglich über $<$ www.zis-online.com>).

3 Verfügung der Generalbundesanwaltschaft, veröffentlicht in JZ 2005, S. 311 f.

4 OLG Stuttgart NStZ 2006, S. 117 ff.

5 Siehe etwa die Besprechungen von Ambos NStZ 2006, S. 434 ff.; Basak in: Institut für Kriminalwissenschaften und Rechtsphilosophie Frankfurt a.M. (Hrsg.), Jenseits des rechtsstaatlichen Strafrechts (2007), S. 499 ff.; Singelnstein/ Stolle ZIS 2006, S. 118 ff.; kritisch auch KMR, Kommentar zur Strafprozessordnung (Plöd), 46. Lfg. (2007), § 153f StPO, Rn.13. 
dächtige, im November 2006 ein zweites Mal einzureichen ${ }^{6}$. Am 5. April 2007 entschied die Generalbundesanwaltschaft erneut, keine Ermittlungen aufzunehmen ${ }^{7}$. Hiergegen soll nun ein zweites Mal ein Klageerzwingungsverfahren nach $\S 172$ StPO betrieben werden ${ }^{8}$.

Der Fall Rumsfeld ist insofern exemplarisch, als die Generalbundesanwaltschaft bislang in fast jedem der mehreren Dutzend Fälle, die nach dem VStGB angezeigt wurden, schon die Aufnahme von Ermittlungen verweigert hat ${ }^{9}$ und damit bisher diesem Gesetz durch völlige Nichtanwendung entgegen seiner ambitionierten Intention zur völligen praktischen Bedeutungslosigkeit verhilft ${ }^{10}$. Dass dies genauso gewollt ist, zeigen die teilweise sehr fragwürdigen Begründungen, mit denen die Einleitung von Ermittlungen abgelehnt werden ${ }^{11}$.

Dieser Text soll nach einer Schilderung des Sachverhaltes und dessen materiellrechtlicher Würdigung (A.) vor allem die prozessualen Begründungen genauer beleuchten, mit denen die Generalbundesanwaltschaft bisher Ermittlungen verweigert (B.). Dass diese im Ergebnis nicht mit Wortlaut und Systematik des Gesetzes oder dem Willen des Gesetzgebers übereinstimmen, führt zwar zu einem klaren rechtlichen Ergebnis, dessen praktische Durchsetzungsfähigkeit gegen politische Opportunitäten sich in den anstehenden Gerichtsverfahren aber erst zeigen musS. Allerdings sollten die Beteiligten an diesen Verfahren in Bezug auf das VStGB im Auge behalten, dass ein Gesetz, das erfolgreich von den zuständigen Strafverfolgungsbehörden ignoriert werden kann, keine Rechtswirkungen entfaltet und ein (trauriger) Fall toten Rechts wäre.

\section{B. Worum es geht: Die angezeigten Sachverhaltskomplexe und ihre rechtliche Bewertung}

Gegenstand der zweiten Anzeige sind im Unterschied zum ersten Versuch nun nicht nur die Gräuel von Abu Ghraib im Irak, sondern auch der Umgang mit Gefangenen im

6 Diese zweite Anzeige ist zugänglich unter <www.ccr-ny.org/v2/GermanCase2006/Docs/ Complaint_German_vol1.pdf> und <www.ccr-ny.org/v2/GermanCase2006/Docs/Complai nt_German_vol2.pdf>.

7 Diese Verfügung liegt in einer englischen Übersetzung unter <http://www.ccr-ny.org/v2/ GermanCase2006/Docs/prosecutorsdecision.pdf> vor.

8 Der entsprechende Klageerzwingungsantrag zu Az. 3 ARP 156/06-2 der Generalbundesanwaltschaft liegt dem Autor dieses Textes als Entwurf vor und kann vom federführenden Rechtsanwalt Kaleck über <www.diefirma.net> bezogen werden.

9 Nach BT-DruckS. 16/2692 erfolgte bis zum 30.06.2006 auf 58 Strafanzeigen nach dem VStGB die Eröffnung eines einzigen Ermittlungsverfahrens bezüglich einer Rebellengruppe im Kongo. In den übrigen 57 Fällen wurde bereits der Eintritt in das Ermittlungsverfahren verweigert.

10 Kaleck/Wiener, Normen der inter-nationalen Politik (2006), S. 16 ff., zugänglich unter <www.dvpw.de/fileadmin/docs/2006xWieKal.pdf>. Hierzu fand am 24.10.2007 eine Expertenanhörung im Bundestagsausschuss für Menschenrechte und humanitäre Hilfe statt, siehe <www.bundestag.de/aktuell/hib/2007/2007_269/01.html>.

11 Instruktiv ist hier etwa der Fall des schwerer Menschenrechtsverletzungen verdächtigten usbekischen Innenministers Almatow, der sich für eine medizinische Behandlung in Deutschland befand, gegen den aber der Generalbundesanwalt unter Verweis auf § 153f StPO kein Ermittlungsverfahren einleitete (Pressemitteilung 9/2006 des Generalbundesanwaltes vom 31.03.2006). Siehe dazu etwa die Kleine Anfrage von Abgeordneten der FDP-Fraktion des Bundestages vom 18.05.2006 (BT-DruckS. 16/1579) und die Antwort der Bundesregierung hierauf (BT-DruckS. 16/1781). Kritisch hierzu etwa Zappalà Journal of International Criminal Justice (JICJ) 4 (2006), S. 602 ff. 
Lager in Guantànamo Bay, Kuba, durch die US-Armee ${ }^{12}$. Beide Komplexe des Sachverhaltes sollen kurz in Erinnerung gerufen werden (I.), bevor sie einer materiellrechtlichen Würdigung unterzogen werden (II.).

\section{Die angezeigten Sachverhalte}

»Abu Ghraib« ist drei Jahre nach Veröffentlichung der grausamen Bilder von der Misshandlung irakischer Gefangener im April 2004 im Fernsehsender CBS zu einem Synonym für Folter und Erniedrigung geworden. Menschen wurden geschlagen und getreten, sexuell entwürdigt und psychisch gequält, und all dies wurde fotografisch dokumentiert. Die Bilder etwa von dem mit einer Kapuze über dem Kopf auf eine Holzkiste gestellten Mann, dessen Hände mit Stromkabeln verbunden wurden, verbunden mit der Ankündigung, er werde von Stromstößen getötet, wenn er von der Kiste falle, oder von dem von einer weiblichen Aufseherin an einer Hundeleine am Hals gehaltenen nackten männlichen Gefangenen, sind in die globale Ikonographie als Sinnbilder für amerikanische Menschenrechtsverletzungen eingegangen ${ }^{13}$.

Der im Februar 2004 verfasste Report des die Zustände in Abu Ghraib untersuchenden Generalmajors Taguba ${ }^{14}$ betrachtet unter anderem als bewiesene Taten: Schläge; Tritte; den Einsatz von Hunden als Drohung wie zur Zufügung schwerer Bissverletzungen; Vergewaltigung und verschiedene sexuelle Nötigungen, von erzwungener teilweise tagelanger Nacktheit bis zu erzwungenen Film- und Fotoaufnahmen in sexuell eindeutigen Posen; Bedrohung mit geladenen Schusswaffen; Quälereien der Gefangenen mit kaltem Wasser oder den phosphorhaltigen Inhaltsstoffen von Leuchtstoffröhren. $\mathrm{Zu}$ diesen Vorwürfen sind seit dem viele weitere Details bekannt geworden ${ }^{15}$, die allerdings an der grundsätzlichen rechtlichen Bewertung wenig ändern.

Während die Vorgänge als solche auch von der Regierung der Vereinigten Staaten von Amerika nicht geleugnet werden, lief allerdings die als beendet zu betrachtende strafrechtliche Aufarbeitung dieser Untaten auf die durch die US-Regierung immer betonte Behauptung hinaus, hier habe es sich um Exzesstaten einzelner Aufseher gehandelt $^{16}$. So betraf die Strafverfolgung weitestgehend keine militärisch oder politisch Vorgesetzten, sondern nur die unmittelbar an den durch die Fotos unzweifelhaft dokumentierten Misshandlungen beteiligten Militärpolizisten, von denen einige wenige

12 Anschaulich zu den Zuständen in Guantànamo die Beschreibung von Kaiser SPIEGEL 33/2007, S. 56 ff., der die Geschichte des Selbstmordes dreier Häftlinge dieses Lagers nachzeichnet.

13 Eine große Zahl von 2006 veröffentlichten weiteren Bildern aus Abu Ghraib ist zugänglich unter <www.salon.com/news/abu_ghraib/2006/03/14/introduction/index.html>.

14 Zugänglich unter <www.npr.org/iraq/2004/prison_abuse_report.pdf $>$, im Folgenden als Taguba-Report bezeichnet.

15 Hingewiesen sei hier etwa auf den Report von Fay und Jones vom August 2004, zugänglich unter <news.findlaw.com/hdocs/docs/dod/fay82504rpt.pdf>, von dem ein Teil für die Strafanzeige von 2006 übersetzt wurde, dort S. 146-188.

16 Siehe etwa Pitzke vom 31.07.2007 unter <www.spiegel.de/politik/ausland/0,1518,503182, 00.html> auch zu dem zuletzt beendeten Prozess gegen den damaligen Leiter der Verhörabteilung von Abu Ghraib Jordan, der wegen Verfahrensfehlern nur wegen Ungehorsams verwarnt wurde. 
zu längeren Haftstrafen verurteilt, die meisten aber durch Militärgerichte mit begrenzter Strafgewalt nur sehr milde bestraft wurden ${ }^{17}$. Völkerstrafrechtliche Tatbestände spielten hierbei keinerlei Rolle.

Dagegen wurde schon im bereits erwähnten Taguba-Report wie auch in zahlreichen Aussagen Beteiligter nahe gelegt, dass diese Misshandlungen der Gefangenen dazu dienen sollten, diese zur Kooperation bei ihren Verhören zu zwingen ${ }^{18}$. In einer ganzen Reihe von Dokumenten aus dem Verteidigungsministerium und seitens höchster Rechtsberater der Regierung wird mehr als deutlich, dass es systematische Strategie der USA im Rahmen des sogenannten Kriegs gegen den Terror war, Gefangene durch gravierende Übelszufügung zu Aussagen zu bewegen ${ }^{19}$. Durch Dokumente, deren Veröffentlichung Menschenrechtsgruppen in den USA mit Klagen nach dem Freedom of Information-Act erzwungen haben, ist belegt, dass nach den vom damaligen Verteidigungsminister Rumsfeld selbst abgesegneten Richtlinien zum Umgang mit Gefangenen z.B. das Verharrenlassen in schmerzhaften Körperpositionen über längere Zeiträume, Schlafentzug, etwa durch ununterbrochene Beschallung mit lauter Musik, Nahrungsentzug, Bedrohungen etc. als von höchster Stelle genehmigte Methoden zur Erlangung von Informationen eingesetzt werden sollten und auch wurden ${ }^{20}$.

Diese letztgenannten Methoden fanden auch Verwendung im Gefangenenlager Guantànamo $^{21}$, dessen Name inzwischen ebenfalls weltweit als Synonym für die Rechtlosstellung Gefangener durch die USA gilt. In diesem Lager halten die USA Menschen fest, die in den verschiedenen Operationen in ihrem selbst so bezeichneten »Krieg gegen den Terror« gefangen genommen wurden. Die Insassen sind überwiegend in Afghanistan und Pakistan aufgegriffen worden. Die Regierung der Vereinigten Staaten von Amerika verweigert diesen Menschen die Anerkennung als Kriegsgefangene sondern bezeichnet sie als »unlawful « oder »illegal combatants « und nahm das Recht in Anspruch, sie beliebig lange ohne auch nur die Möglichkeit gerichtlicher

17 Eine Zusammenstellung der ergangenen Urteile enthält die Strafanzeige von 2006 (Fn. 6), S. 33 ff. Selbst der zu einer Haftstrafe von acht Jahren verurteilte Ivan Frederick ist nun nach weniger als der Hälfte der Haftzeit auf Bewährung entlassen worden, siehe die Meldung vom 01.10.2007 unter <www.news365.de/content/view/16882/1890/>.

18 Taguba-Report (Fn. 14), S. 18; Report des Internationalen Kommittees vom Roten Kreuz vom Februar 2004 (ICRC-Report), S. 12 (zugänglich unter <www.cbsnews.com/htdocs/ pdf/redcrossabuse.pdf $>$ ).

19 Eine Zusammenstellung dieser Dokumente findet sich etwa unter <www.washingtonpost. com/wp-dyn/articles/A62516-2004Jun22.html>; siehe auch Hentoff in der online-Ausgabe von The Village Voice vom 28.08.2007 unter <www.villagevoice.com/news/0735, hentoff, 77643,6.html>.

20 Berühmt geworden ist hier vor allem ein Memo von General Counsel Haynes vom 27.11.2002 über Verhörmethoden, auf dem Rumsfeld handschriftlich zum erzwungenen Stehen von Gefangenen vermerkte: »However, I stand for 8-10 hours a day. Why is standing limited to 4 hours? " (unter <www.washingtonpost.com/wp-srv/nation/documents/ dodmemos.pdf $>$. Zu von der CIA angewandten Methoden, die ebenfalls vom Weißen Haus genehmigt gewesen sein sollen, siehe Hentoff (Fn. 19).

21 Dazu die Strafanzeige von 2006 (Fn. 6), S. 121 ff. und bezogen auf den konkreten Anzeigeerstatter S. 190 ff.; siehe auch Bassiouni Case Western Reserve Jounal of International Law (CWRJIL) 37 (2006), S. 389 ff. 
Kontrolle der Haft festzuhalten und zu verhören ${ }^{22}$. Zwischenzeitlich wurde ein großer Teil der Insassen entlassen, denen nach meist mehrjähriger Haft bestätigt wurde, weder Terroristen zu sein, noch Verbrechen gegen die USA verübt zu haben oder eine Gefahr für ihre Bürger darzustellen ${ }^{23}$.

Die fehlende Möglichkeit einer gerichtlichen Überprüfung der Inhaftierung wurde für Guantànamo in mehreren aufsehenerregenden Entscheidungen des Supreme Court als rechts- und verfassungswidrig gekennzeichnet und die Regierung der USA dazu gezwungen, eine gerichtliche Überprüfung der Haft zuzulassen ${ }^{24}$. Dies hat die Regierung Bush nunmehr Militärgerichten übertragen, vor denen allerdings die Rechte der Betroffenen nach wie vor stark eingeschränkt sind.

Abgesehen davon, dass die US-Regierung hier Menschen außerhalb jeder rechtlichen Kontrolle in Haft hält, die zudem ohne Benachrichtigung ihrer Angehörigen über ihren Verbleib um die halbe Welt geflogen wurden, steht auch die Behandlung der Gefangenen in Guantànamo weltweit in der Kritik. Anfangs wurden die Gefangenen in Maschendrahtkäfigen im Freien festgehalten. Inzwischen wurde zwar Gebäude errichtet, dennoch kann hier nicht von einem regulären Haftbetrieb gesprochen werden. Es gibt glaubhafte Berichte über sexuelle und religiöse Demütigungen sowie Misshandlungen verschiedenster $\mathrm{Art}^{25}$. Aber auch die Inhaftierung ohne jeden Kontakt zur AuBenwelt und ohne jede Aussicht auf Entlassung ist für die Betroffenen nach Berichten ehemaliger Insassen psychisch eine sehr schwere Belastung ${ }^{26}$.

\section{Die rechtliche Würdigung dieser Sachverhalte}

Die vorläufige rechtliche Bewertung dieser Sachverhalte soll in zwei Schritten erfolgen. Zunächst soll dargelegt werden, welche Tatbestände hier in Betracht kommen (1.), dann soll die Zurechnung dieser Taten an die hier angezeigten militärischen und zivilen Vorgesetzten gesondert thematisiert werden.

\section{Die relevanten Tatbestände}

Für die hier besprochene Strafanzeige an die Bundesanwaltschaft kommen als Grundlage für eine Bestrafung die Tatbestände des seit nunmehr fünf Jahren existierenden VStGB in Betracht. Diese Umsetzung des durch das Rom-Statut des ICC kodifizierten

22 Siehe etwa den Rechtsberater im US-Außenministerium Bellinger German Law Journal (GLJ) 8 (2007), S. 871 (875 ff.).

23 Der hierzulande prominenteste Fall ist der von Murat Kurnaz, der seine Erlebnisse unter dem Titel »Fünf Jahre meines Lebens« (2007) veröffentlicht hat. Eine Chronologie des

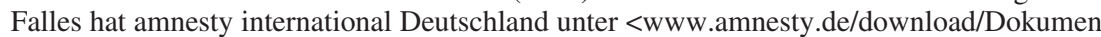
tationMuratKurnaz.pdf > veröffentlicht.

24 Siehe vor allem die Entscheidung des Supreme Court der USA vom 28.04.2004 im Fall Rasul vS. Bush (<www.ccr-ny.org/v2/reports/docs/Rasul\%20Decision.pdf>) sowie die am gleichen Tag ergangenen Entscheidungen in den Fällen Hamdi und Padilla, dazu auch Aldrich JICJ 2 (2004), S. 967 ff.; Fletcher JICJ 2 (2004), S. 953 ff. Zum am 29.06.2006 entschiedenen Fall Hamdan vS. Rumsfeld siehe Fletcher JICJ 4 (2006), S. 442 ff.

$25 \mathrm{Vgl}$. die Strafanzeige von 2006 (Fn. 6), S.15.

26 Hierzu etwa Kaiser SPIEGEL 33/2007, S. 56 ff. 
Völkerstrafrechts in deutsches Recht gilt ausweislich § 1 VStGB auch für Auslandstaten, bei denen weder Täter noch Opfer Deutsche sind. § 1 VStGB postuliert hier das Weltrechtsprinzip ausdrücklich auch ohne weitergehenden Inlandsbezug und ist hier weiter als der insoweit verdrängte $\S 6 \mathrm{StGB}^{27}$.

Damit rückt vor allem $\S 8$ VStGB in den Mittelpunkt des Interesses, also die Kriegsverbrechen gegen Personen. Voraussetzung für die Anwendbarkeit dieser Norm ist zunächst ein Zusammenhang mit einem bewaffneten Konflikt ${ }^{28}$. Dieser ist im Falle des Irak unzweifelhaft gegeben, weil auch die andauernde Besatzung eines Landes durch fremde Streitkräfte als bewaffneter Konflikt im Sinne dieser Norm anzusehen ist ${ }^{29}$. Eine solche lag bei den Fällen in Abu Ghraib vor, weil im fraglichen Zeitraum (Ende 2003 bis April 2004) die unter der Führung der USA agierende »Koalition der Willigen« die Verwaltung des Irak noch vollständig inne hatte ${ }^{30}$. Auch ein funktionaler Zusammenhang zwischen diesem Besatzungsregime und den Misshandlungen liegt unzweifelhaft vor, weil die Misshandelten zu Zwecken der militärischen Aufklärung verhört werden sollten und nur deswegen interniert waren ${ }^{31}$. Da es sich um einen internationalen Konflikt handelte, waren die in Abu Ghraib gefangenen Personen auch durch das humanitäre Völkerrecht geschützt im Sinne des $\S 8$ Abs. 6 VStGB, und zwar entweder als Zivilisten ( $\$ 8$ Abs. 6 Nr. 1 VStGB) oder als Angehörige der gegnerischen Streitkräfte, die nach ihrer Gefangennahme hilflos waren ( 8 Abs. 6 Nr. 3 VStGB $)^{32}$. Damit ist der Tatbestand des $\S 8$ VStGB auf Misshandlungen dieser Gefangenen anwendbar.

Ebenso deutlich ist die Anwendbarkeit dieser Norm auf den hier zur Anzeige gebrachten Fall aus Guantànamo. Der Anzeigeerstatter wurde im Zuge der Invasion Afghanistans durch alliierte Truppen im Grenzgebiet zwischen Afghanistan und Pakistan als mutmaßlich hochrangiges Mitglied von Al-Qaida festgenommen und nach Guantànamo Bay verbracht. Die Invasion und nachfolgende Besetzung Afghanistans stellte zweifelsohne einen internationalen Konflikt dar, in dessen Zuge die Verhaftung des

27 BR-DruckS. 29/02, S. 29; in diesem Sinne auch Ambos, Internationales Strafrecht (2006), § 3 Rn.102; Satzger, Internationales und Europäisches Strafrecht (2005), § 16, Rn. 38, und NStZ 2002, S. 125 (131 f.); siehe auch schon zuvor Lagodny/Nill-Theobald JR 2000, S. 205 (206).

28 Triffterer (Fenrick), Commentary on the Rome Statute of the International Criminal Court (1999), Art. 8 ICC-Statut, Rn.2.

29 Dazu etwa die Elements of Crime zu Art. 8 ICC-Statut, S. 15, Fn.34, die entsprechend Art. 9 ICC-Statut von der Vertragsstaatenversammlung beschlossene amtliche Kommentierung der einzelnen Tatbestände des ICC-Statuts (unter <www.icc-cpi.int/library/about/officialjournal/Element_of_Crimes_English.pdf>). Siehe auch Ambos (Fn. 27), § 7 Rn. 231; Satzger (Fn. 27), § 15 Rn.61 ff.; Werle, Völkerstrafrecht (2003), Rn. 815 ff., insb. 823.

30 Basak HuV-I 2005, S. 85 (87).

31 Vgl. dazu die Strafanzeige von 2004 (Fn. 2), S. 82; siehe auch ICTY, Beschluss der Appeals Chamber im Fall Tadić vom 02.10.1995, Ziff.70; ICTY, Urteil der Appeals Chamber im Fall Kunarac vom 12.06.2002, Ziff. 59; zur Notwendigkeit eines solchen Zusammenhangs auch BRDrucks.29/02, S. 55 f.; Satzger (Fn. 27), § 15 Rn.58; Werle (Fn. 29), Rn.837 ff.

32 Dies entspricht Art. 4 Abs. 1 der IV. Genfer Konvention (Zivilisten) bzw. Art. 4 A der III. Genfer Konvention (Kriegsgefangene). Siehe hierzu auch Ambos (Fn. 27), § 7 Rn.240; Werle (Fn. 29), Rn.852 ff. 
Anzeigeerstatters erfolgte ${ }^{33}$. Damit gehört er unter den Begrifflichkeiten des $\S 8$ Abs. 6 VStGB auch zu den nach dem humanitären Völkerrecht geschützten Personen. Entweder sind die Kämpfer und Mitglieder von Al-Qaida als Kriegspartei an der Seite des Taliban-Regimes anzusehen ${ }^{34}$, dann ist der Anzeigeerstatter Kriegsgefangener im Sinne des $\S 8$ Abs. 6 Nr. 3 VStGB. Oder sie werden nicht als Teil der regulären Truppen des Kriegsgegners verstanden, dann aber sind sie Zivilisten im Sinne des $\S 8$ Abs. 6 Nr. 1 VStGB ${ }^{35}$. Laut Art. 5 (2) der III. Genfer Konvention haben Gefangene bei Zweifeln so lange als Kriegsgefangene zu gelten, bis diese Zweifel von einem »kompetenten « Gericht ausgeräumt sind ${ }^{36}$. Die von der Regierung der USA insbesondere für die in Guantànamo Bay inhaftierten »Terrorverdächtigen« zur Begründung der angeblichen Nichtgeltung der Regeln des Kriegsvölkerrechts eingeführte Kategorie der »ungesetzlichen feindlichen Kombattanten « (unlawful enemy combatants) ${ }^{37}$ führt im humanitären Völkerrecht jedenfalls nicht zur Schutzlosstellung bei Gefangennahme ${ }^{38}$. Dieses zielt gerade auf einen umfassenden Schutz aller Personen, die in den Wirkungsbereich eines bewaffneten Konfliktes geraten ${ }^{39}$. Die Begründung eines angeblichen »rechtsfreien Raumes«, innerhalb dessen die Betroffenen sich auf gar keine Rechte sollen berufen können ${ }^{40}$, sie vielmehr völlig der Willkür und den Interessen der

33 Siehe hierzu Werle (Fn. 29), Rn.817 und 837 ff. Zur Anwendbarkeit des Kriegsrechts in diesem Konflikt, mithin zu seiner Natur als bewaffneter Konflikt, siehe auch Bellinger GLJ 8 (2007), S. 735 (736); stark differenzierend dagegen Sassòli JICJ 2 (2004), S. 96 (98ff.). Sehr kritisch zum Ansatz der US-Regierung, den »War on Terror « insgesamt unter dem Begriff »armed violence " so aufzufassen, dass hier statt »normalem « Strafrecht das Kriegsrecht gelten solle und damit die US-Regierung zwar in dessen Rahmen agieren, gleichzeitig aber nicht dessen Schutzmechanismen greifen lassen will, Stewart JICJ 5 (2007), S. 26 (27 f.).

34 Vgl. Werle (Fn. 29), Rn.854 f.; hiergegen etwa Bellinger GLJ 8 (2007), S. 735 (742) mit dem Argument, Taliban und Al-Qaida-Kämpfer seien keine staatlichen Akteure und damit nicht von der III. Genfer Konvention geschützt; dies bestreitet Vöneky GLJ 8 (2007), S. 747 (753) zumindest für die Taliban, welche die seinerzeit einzige faktisch existierende Armee des Staates Afghanistans bildeten.

35 So auch Art. 4 Abs. 1 des IV. Genfer AbkommenS. Laut Werle (Fn. 29), Rn.857 bezieht sich dessen Schutz ausdrücklich auch auf »Personen, die Waffengewalt anwenden, ohne als Kombattanten dazu berechtigt zu sein«; ähnlich Ambos (Fn. 27), § 7 Rn.241.

36 So auch Vöneky GLJ 8 (2007), S. 747 (754). Ausführlich hierzu Naqvi International Review of the Red Cross (IRRC) 84 (2002), S. $571 \mathrm{ff}$.

37 Siehe Bellinger GLJ 8 (2007), S. 735 (742). Der US-Military Commisions Act vom 17.10.2006 kodifiziert diese Kategorie erstmals und höchst problematisch, dazu Maxwell/ Watts JICJ 5 (2007), S. 19 ff. Kritisch auch Alvarez CWRJIL 37 (2006), S. 175 (180 f.).

38 Vöneky GLJ 8 (2007), S. 747 (753) schlägt hier den passenderen Begriff der »offensive civilians « vor; siehe auch Chlopak Human Rights Brief Vol.9, IsS. 3 (unter <www.wcl.american.edu/hrbrief/09/3guantanamo.cfm>); Haller, Die Grenzen der Solidarität (2002), S. 79 f.; Sassòli JICJ 2 (2004), S. 96 (101); Werle (Fn. 29), Rn. 857.

39 So ausdrücklich bereits Pictet, Commentary, Bd.4, Geneva Convention Relative to the Protection of Civilian Persons in Time of War (1958), S. 51; siehe auch Sassòli JICJ 2 (2004), S. 96 (101 f.).

40 In diesem Sinne etwa Bellinger GLJ 8 (2007), S. 871 (876); darstellend Chlopak (Fn. 38); Harris Journal of National Security Law \& Policy (JNSLP) 1 (2005), S. 409 (410 ff.); Sassòli JICJ 2 (2004), S. 96 (97 f., mit zahlreichen weiteren Nachweisen); siehe auch Steyn International Criminal Law Quarterly (ICLQ) 53 (2004), S. 1 ff., der das Bild von Guantànamo als »Legal Black Hole« prägt; ebenso Fletcher JICJ 2 (2004), S. 121 ff., sowie bezogen auf Abu Ghraib Garraway JICJ 2 (2004), S. 981 ff. 
sie gefangen haltenden Macht ausgeliefert sind, ist weder mit dem Konzept eines humanitären Völkerrechts noch mit der Universalität beanspruchenden Idee der Menschenrechte in Einklang zu bringen ${ }^{41}$. Nach dem humanitären Völkerrechts sind demnach die in Guantànamo Bay Inhaftierten als von diesem Recht geschützte Personen zu betrachten, soweit ihre Gefangennahme in Zusammenhang mit einem bewaffneten Konflikt steht ${ }^{42}$.

Sind damit die Anwendungsvoraussetzungen der Norm und die persönliche Anwendbarkeit auf die Opfer der hier angezeigten Taten nach $\S 8$ Abs. 1 Satz 1 und Abs. 6 VStGB gegeben, fällt die Subsumtion der gut dokumentierten Handlungen gegen die Gefangenen sowohl in Abu Ghraib als auch in Guantànamo unter die einzelnen Tathandlungen des $\S 8$ VStGB nicht schwer.

So erfüllen nicht nur die unmittelbar körperlichen Misshandlungen wie Schläge, Tritte oder Hundebisse den Tatbestand des $\S 8$ Abs. 1 Nr. 3 VStGB. Auch grausame Verhörmethoden wie das sogenannte $»$ water boarding ${ }^{43}$, erzwungenes andauerndes Stehen in Stresspositionen oder langandauernde Fesselungen in unnatürlichen Körperpositionen, Nahrungs- oder Schlafentzug oder der Einsatz extremer Temparaturen erfüllen den Tatbestand der Folter ${ }^{44}$. All diese vom Pentagon bewilligten und sowohl in Guantànamo als auch in Abu Ghraib zur Erzwingung von Aussagen ${ }^{45}$ eingesetzten Methoden führen zu schweren körperlichen Leiden, häufig auch zu langanhaltenden pathologischen Zustandsverschlechterungen der Opfer ${ }^{46}$. Hierunter fallen aber auch psychische Quälereien, welche $\S 8$ Abs. 1 Nr. 3 VStGB auch ausdrücklich als Tathandlung benennt. Dazu zählen etwa schwerwiegende Entwürdigungen wie erzwungene Nacktheit, insbesondere vor Wachpersonal des jeweils anderen Geschlechts, erzwungene sexuelle Erniedrigungen, andauernde Beleidigungen und Beschimpfungen, insbesondere auch auf die Religion der Opfer bezogen, Verhinderung der Religionsausübung,

41 Sassòli JICJ 2 (2004), S. 96; Vöneky GLJ 8 (2007), S. 747 (755) weist zu Recht auf den jedenfalls immer geltenden Minimalstandard des gemeinsamen Art. 3 der Genfer Konventionen hin, der eben grausame und inhumane Behandlungsweisen verbiete und sowohl habeas corpus als auch grundlegende Verfahrensrechte garantiere.

42 Vgl. hierzu Fleck (Hrsg.), Handbuch des humanitären Völkerrechts (Gasser) (1994), Nr. 501; Werle (Fn. 29), Rn.857; Arnold JICJ 2 (2004), S. 999 (1001). Nicht Gegenstand dieses Verfahrens ist die Frage, ob dies auch auf die Internierten zutrifft, die außerhalb der Länder aufgegriffen wurden, die als Konfliktzonen anzusehen sind, namentlich die in Pakistan aufgegriffenen Al-Qaida-Verdächtigen. Soweit hier aber kein internationaler Konflikt vorliegt, wären diese Personen als Zivilisten und damit als »normale« Kriminelle vor ein ziviles Gericht zu stellen, eine dauerhafte Internierung ohne entsprechendes Urteil wäre gerade nicht durch das Kriegsrecht gerechtfertigt und damit menschenrechtswidrig, siehe Sassòli JICJ 2 (2004), S. 96 (105).

43 Eine sehr eindringliche Schilderung dieser Foltermethode enthält die Strafanzeige von 2006 (Fn. 6), S. 10.

44 ICTY, Urteil der Trial Chamber im Fall Kvoèka vom 02.11.2001, Ziff.144; Werle (Fn. 29), Rn.696; zum Einsatz dieser Methoden auch Hentoff (Fn. 19).

45 Siehe dazu oben unter A.I. Damit wird auch das Erfordernis eines über die Schmerzzufügung hinausgehenden Zwecks erfüllt, dazu Werle (Fn. 29), Rn.880.

46 Letztere sind aber nicht konstitutiv für die Bejahung von Folter, siehe ICTY, Urteil der Trial Chamber im Fall Kvoèka vom 02.11.2001, Ziff.148; Werle (Fn. 29), Rn.697. 
Isolationshaft, Verursachung völliger zeitlicher und räumlicher Orientierungslosigkeit zur Erzeugung von Verwirrung und Angst, etwa durch andauernde Dunkel- oder Helligkeitshaft sowie dauernde Beschallung mit lauter Musik (»white noise «) und anderes ${ }^{47}$. Hier werden schwerste Traumata erzeugt. Persönlichkeit und Willen der Opfer sollen gezielt gebrochen werden, um schneller an Informationen zu gelangen. All dies sind ohne jeden Zweifel nach § 8 Abs. 1 Nr. 3 VStGB verbotene grausame und unmenschliche Behandlungen durch Zufügung schweren seelischen und körperlichen Leidens ${ }^{48}$.

Dabei ist zu beachten, dass das hier letztlich an die Genfer Konventionen angelehnte VStGB anders als die Anti-Folter-Konvention (Convention Against Torture - CAT ${ }^{49}$ ) kein amtliches Handeln fordert, soweit eine Zurechnung an eine durch die Genfer Konventionen gebundene Konfliktpartei vorliegt ${ }^{50}$ - hier die USA. Auch unterscheidet das VStGB - ebenso wie Art. 8 Abs. 2 a) ii) ICC-Statut - ausdrücklich nicht zwischen Folter und unmenschlicher Behandlung, die beide gleichermaßen unter Strafe gestellt werden $^{51}$. Versuche einer diesbezüglichen Differenzierung durch Rechtsberater der US-Regierung laufen - unabhängig von ihrer inhaltlichen Unvertretbarkeit - in Bezug auf die Begründung einer nicht gegebenen Strafbarkeit nach Völkerstrafrecht leer ${ }^{52}$.

Unstreitig erfüllen die in mehreren veröffentlichten Zeugenaussagen und Untersuchungsberichten erwähnten sexuellen Übergriffe auf Gefangene insbesondere in Abu Ghraib auch den den Tatbestand des $\S 8$ Abs. 1 Nr. 4 VStGB. Die im Taguba-Report als bewiesen angesehene Vergewaltigung einer weiblichen Gefangenen durch einen Militärpolizisten fällt ebenso hierunter wie die anale Penetration von Gefangenen mit Gegenständen $^{53}$. Auch das Zwingen nackter Gefangener in sexuell eindeutige Posen, das fotografisch dokumentiert ist ${ }^{54}$, erfüllt den Tatbestand der sexuellen Nötigung ${ }^{55}$.

Bezüglich der jahrelangen Inhaftierungen in Guantànamo Bay, etwa bei dem hier als Anzeigeerstatter auftretenden A., der dort seit annähernd fünf Jahren gefangen gehalten wird, ist zudem an $\S 8$ Abs. 1 Nr. 7 VStGB zu denken, nach dem die Verhängung und Vollstreckung einer erheblichen Strafe ohne unparteiisches Gerichtsverfahren ein Kriegsverbrechen ist $^{56}$. Dies gilt zumindest dann, wenn er als Al-Qaida-Mitglied nicht als regulärer Kombattant und damit als Kriegsgefangener angesehen wird ${ }^{57}$, was die USA explizit verweigern ${ }^{58}$.

47 Siehe auch Werle (Fn. 29), Rn.883.

48 Basak HuV-I 2005, S. 85 (87 f.); siehe auch Triffterer (Fenrick) (Fn. 28), Art. 8 ICC-Statut, Rn.10 ff.; Satzger (Fn. 27), § 15 Rn.46.

49 Convention against Torture, vom 10. Dezember 1984, BGBl. 1990 II 246.

50 Satzger (Fn. 27), § 15 Rn.46; Werle (Fn. 29), Rn.879.

51 Werle (Fn. 29), Rn.894 f.

52 Siehe auch Alvarez CWRJIL 37 (2006), S. 175 (182 ff.).

53 Werle (Fn. 29), Rn.706; zu diesen Taten siehe Taguba-Report (Fn. 14), S. 17.

54 Werle (Fn. 29), Rn.716. Siehe etwa die Sammlung unter der in Fn. 13 genannten Adresse unter den Stichworten »Sexual Exploitation« und »Dog Pile«.

55 Siehe $\S \S 177$, 184f StGB; Basak HuV-I 2005, S. 85 (88).

56 Dazu Werle (Fn. 29), Rn.923 ff.

57 Siehe Satzger (Fn. 27), § 15 Rn.69. Kriegsgefangene dürfen ohne Urteil bis zum Ende der Feindseligkeiten gefangen gehalten werden, genießen dann aber auch den vollen Schutz der III. Genfer Konvention.

58 Bellinger GLJ 8 (2007), S. 735 (739); Chlopak (Fn. 38); Sassòli JICJ 2 (2004), S. 96 (104); Vöneky GLJ 8 (2007), S. 747 (753). 
Zwar nehmen die USA für sich in Anspruch, dass die Häftlinge auf Guantànamo Bay dort nur präventiv festgehalten würden, um sie an der Begehung von Terrorakten gegen Bürger der Vereinigten Staaten von Amerika oder an Kriegsakten gegen US-Truppen zu hindern $^{59}$. Aber dies ändert nichts an der Tatsache, dass eine fünfjährige Haft und deren unbefristetes weiteres Andauern ${ }^{60}$ Strafcharakter hat. Diese Praxis des Wegsperrens ohne Gerichtsurteil und ohne auch nur den Zugang zu einem gerichtlichen Verfahren ist in den Leitentscheidungen des Supreme Court der USA zu Guantànamo bereits nach US-Verfassungsrecht als rechtswidrig gekennzeichnet und die Regierung dazu verpflichtet worden, den Inhaftierten die gerichtliche Überprüfung ihrer Haftgründe vor einem rechtsstaatlichen Grundsätzen genügenden Gericht zu ermöglichen ${ }^{61}$. Allein die Lektüre dieser Urteile belegt, dass hier diese Mindeststandards des habeas corpus und eines rechtsstaatlichen Verfahrens eben nicht eingehalten wurden ${ }^{62}$. Daran ändert auch die von der US-Regierung durchgesetzte Einführung des Military Commissions Act (MCA) nichts, in dem unter anderem die Einsetzung von Militärkommissionen als gerichtliche Prüfungsinstanzen für die Haftgründe festgelegt werden ${ }^{63}$. Erstens erscheint es sehr zweifelhaft, ob diese Instanzen als »unparteiische Gerichte« im Sinne des $\S 8$ Abs. 1 Nr. 7 VStGB angesehen werden können ${ }^{64}$. Zweitens aber macht gerade diese durch den Supreme Court erzwungene Gesetzgebung sehr deutlich, dass bis zum heutigen Tage in Guantànamo Menschen ohne einen rechtsstaatlichen Mindeststandards genügenden Prozess über viele Jahre unter extremen Bedingungen gefangen gehalten werden, und dies wegen des im MCA fehlenden Rechts auf einen schnellen Prozess auch auf unabsehbare Zeit so bleiben wird ${ }^{65}$. Insofern erscheint auch der Tatbestand des $\S 8$ Abs. 1 Nr. 7 VStGB erfüllt.

Weiter erfüllen die in Abu Ghraib und Guantànamo erfolgten gut dokumentierten verschiedenen Methoden der Be-, oder eher Misshandlung der Gefangenen den Auffangtatbestand des $\S 8$ Abs. 1 Nr. 9 VStGB, der schwerwiegende Entwürdigungen und Erniedrigungen unter Strafe stellt ${ }^{66}$. Hierunter fallen die auf den veröffentlichten Bil-

59 Bellinger GLJ 8 (2007), S. 735 (737 und 742).

60 Letzteres verteidigt Bellinger GLJ 8 (2007), S. 735 (743).

61 Siehe etwa die in Fn. 24 genannten Fälle.

62 Fletcher JICJ 4 (2006), S. 442 (444).

63 Unter Berufung auf diese inzwischen eingerichtete Möglichkeit, gegen die sich die Regierung Bush jr. lange gewehrt hatte, nimmt Bellinger EJIL 8 (2007), S. 735 (742 ff.) für diese Regierung nun in Anspruch, einen anspruchsvollen rechtlichen Rahmen für die Internierung, Befragung und gerichtliche Behandlung der in Guantànamo Gefangenen errichtet zu haben.

64 Sehr kritisch hierzu etwa Dorf JICJ 5 (2007), S. 10 ff.; Meyer JICJ 5 (2007), S. 48 ff. (zu prozessrechtlichen Unzulänglichkeiten dieser Gerichte); Stewart JICJ 5 (2007), S. 26 (29 ff.). In den USA ist die Frage, ob diese Militärkommissionen den dortigen verfassungsrechtlichen Vorgaben genügen, Gegenstand des laufenden Verfahrens Al Odah vS. Bush vor dem Supreme Court. Siehe dazu auch die veröffentlichten Quellen unter <www.ccrny.org/v2/legal/september_11th/sept11 Article.asp?ObjID=LC1mo1eLnN\&Content=971>.

65 Meyer JICJ 5 (2007), S. 48 (54).

66 Siehe hierzu etwa Werle (Fn. 29), Rn.910 f.; Sadat ASIL Insight May 2004 (zugänglich unter $<$ www.asil.org/insights/insigh134.htm>) weist darauf hin, dass hierunter schon das Zurschaustellen von nach dem humanitären Völkerrecht geschützten Personen sowie erzwungene Foto- oder Videoaufnahmen fallen, worauf sich auch Präsident Bush jr. und Verteidigungsminister Rumsfeld berufen haben, als sie ihrer Empörung über einen Bericht über US-amerikanische Kriegsgefangene im irakischen Staatsfernsehen im März 2003 Ausdruck verliehen und mit einer Verfolgung der Verantwortlichen als Kriegsverbrecher drohten. 
dern aus Abu Ghraib dokumentierten Grausamkeiten und Inszenierungen entwürdigter Gefangener ebenso wie die aus Guantànamo immer wieder berichteten Methoden religiöser Verächtlichmachung oder sexueller Erniedrigung, die als »Strafmaßnahme« gegen unkooperative Häftlinge eingesetzt wurden.

Soweit die Gefangenen, ob in Abu Ghraib oder Guantànamo, Zivilisten waren, also keine Kämpfer der gegnerischen Konfliktparteien, greift zudem $\S 8$ Abs. 3 Nr. 1 VStGB, der die unrechtmäßige, also ohne hinreichende Rechtsgrundlage erfolgende, Inhaftierung von Zivilpersonen als Kriegsverbrechen unter Strafe stellt ${ }^{67}$. Liest man hierzu etwa den Bericht des Internationalen Kommittees vom Roten Kreuz, nachdem zu der fraglichen Zeit 70-90 \% der Gefangenen in Abu Ghraib zu Unrecht verhaftet worden waren ${ }^{68}$, liegt die Annahme dieser Norm nahe ${ }^{69}$.

Die Anzeige selbst geht nicht auf $\S 7$ VStGB ein, obwohl diese Norm ebenfalls die Folter von Inhaftierten (Abs. 1 Nr. 5), Sexualstraftaten (Abs. 1 Nr. 6) sowie das sowohl bezüglich der Gefangenen in Abu Ghraib als auch der Insassen von Guantànamo nicht fernliegende Verschwindenlassen von Personen (Abs. 1 Nr. 7$)^{70}$ als Verbrechen gegen die Menschlichkeit unter Strafe stellt. Problematisch ist hier die Grundvoraussetzung dieser Norm nach Abs. 1, also der ausgedehnte oder systematische Angriff auf die Zivilbevölkerung ${ }^{71}$. Allerdings können die in dem bereits erwähnten ICRC-Report genannten Zahlen an Gefangenen und an unschuldig Festgehaltenen zumindest als Anlass dienen, die schwierige Abgrenzungsfrage, wann denn ein Angriff hinreichend ausgedehnt ist, um von einem Verbrechen gegen die Menschlichkeit zu sprechen, einmal aufzuwerfen ${ }^{72}$.

Bezüglich Guantànamo stellt sich die Frage, gegen wen sich denn ein ausgedehnter oder systematischer Angriff hätte richten können, denn die dort Internierten entstammen keiner geographisch oder durch sonstige gemeinsame Merkmale gekennzeichneten unterscheidbaren Personengruppe ${ }^{73}$, außer dass es weltweit verhaftete, in den Augen der US-Behörden des Terrors Verdächtige sind. Geht man nach den sonst üblichen Begrifflichkeiten des humanitären Völkerrechts davon aus, dass die in Konfliktgebieten Gefangenen, wie auch der hiesige Anzeigeerstatter, zumindest weit überwiegend als Kombattanten in einem bewaffneten Konflikt anzusehen sind, können diese nicht

67 Werle (Fn. 29), Rn.937 f. verdeutlicht, dass selbst wenn eine Verhaftung wegen einer Bedrohung einer Konfliktpartei durch einen Zivilisten erfolge, diese dann rechtswidrig werde, wenn diesem, wie hier geschehen, die prozessualen Rechts aus der IV. Genfer Konvention verweigert würden.

68 ICRC-Report (Fn. 18), S. 8.

69 Strafanzeige von 2004 (Fn. 2), S. 40 f.

70 Dazu Ambos (Fn. 27), § 7 Rn. 216 f.

71 Ambos (Fn. 27), § 7 Rn.182 ff.; Satzger (Fn. 27) §15 Rn.34 ff.; Werle (Fn. 29), Rn.628 ff.; siehe auch Triffterer (Dixon) (Fn. 28), Art. 7 ICC-Statut, Rn.88.

72 Basak HuV-I 2005, S. 85 (88 f.).

73 Ambos (Fn. 27), § 7 Rn.189. 
als Opfer dieser Norm angesehen werden ${ }^{74}$, so dass $§ 7$ VStGB zumindest für diesen Komplex des hier gegenständlichen Verfahrens nicht in Betracht kommt ${ }^{75}$.

Es kann festgehalten werden, dass zumindest bezüglich der Begehung von Kriegsverbrechen nach $\S 8$ VStGB in den oben geschilderten Modalitäten mehr als nur ein Anfangsverdacht besteht. Die geschilderten Sachverhalte sind bestens dokumentiert und auch in verschiedenen amtlichen Berichten der Behörden der Vereinigten Staaten aufgearbeitet, so dass diese als sicher beweisbar unterstellt werden können. Die rechtliche Bewertung dieser Sachverhalte nach dem VStGB unterliegt ebenfalls keinen ernsthaften Zweifeln, so dass insoweit nicht nur ein Anfangsverdacht, sondern durchaus ein hinreichender Tatverdacht im Sinne des $\S 170$ Abs. 1 StPO besteht ${ }^{76}$.

\section{Die Beteiligung der Angezeigten}

Die genannten Tatbestände wurde durchweg nicht von den Angezeigten eigenhändig verwirklicht. Ein Verdacht gegen die hier angezeigten militärischen und zivilen hochrangigen Vorgesetzten, der zumindest die Aufnahme von Ermittlungen einfordert ( 152 Abs. 2 StPO), wenn er nicht bereits eine Klageerhebung trägt ( 170 Abs. 1 StPO), kann aber auch auf indirekteren Zurechnungsmechanismen beruhen.

\section{a) Beteiligungsformen}

In Betracht kommt hier zunächst die Figur der mittelbaren Täterschaft in Form der Organisationsherrschaft ${ }^{77}$, die auch vom BGH seit der Politbüroentscheidung auf Vorgesetzte wegen Straftaten von Untergebenen angewendet wurde ${ }^{78}$. Vor allem bei den un-

74 Denn dann sind sie jedenfalls keine Zivilbevölkerung mehr, vgl. Satzger (Fn. 27) § 15 Rn.36; Werle (Fn. 29), Rn.630.

75 Für andere Insassen von Guantànamo mag sich diese Frage dennoch stellen. Gemeint sind hier Personen, die gerade nicht in offenen bewaffneten Konflikten gefangen genommen wurden, sondern in Drittländern im Rahmen dort durchgeführter Antiterroreinsätze inhaftiert wurden und dann von den USA entweder in sogenannte »Black Sites «, deren Existenz inzwischen von Präsident Bush jr. selbst bestätigt wurde, verbracht oder eben auch in Guantànamo interniert wurden; siehe hierzu etwa die Dokumentation des ebenfalls vom CCR betreuten Falles Khan unter $<$ www.ccr-ny.org/v2/reports/report.asp?ObjID=v1VoZ8 dNfi\&Content=858>. Nimmt man einerseits die martialische Rhetorik der Regierung der Vereinigten Staaten von einem »Krieg gegen den Terror « ernst, und konstatiert andererseits, dass zumindest das Völkerrecht keine Kategorie des feindlichen Terroristen kennt, sondern den Terrorismus als letztlich ziviles Verbrechen einstuft, dann kann angesichts der Intensität der Maßnahmen der US-Regierung der Begriff des »systematischen Angriffs auf eine Zivilbevölkerung « zumindest vertretbar bejaht werden.

$76 \mathrm{Zu}$ diesem Begriff siehe etwa Karlsruher Kommentar zur Strafprozessordnung (KK) (Schmid), 5.Aufl. (2003), § 170 StPO, Rn.3; Meyer-Goßner, Strafprozessordnung, 50. Aufl. (2007), § 170 StPO, Rn.1.

77 Hierzu Leipziger Kommentar Strafgesetzbuch (Schünemann) (LK), 12.Aufl. (2007), § 25 StGB, Rn.122 ff.; Schönke/Schröder (Sternberg-Lieben), Strafgesetzbuch, 27.Aufl. (2006), \$25 StGB, Rn.25 f.; zu deren Anerkennung im Völkerstrafrecht siehe auch Werle (Fn. 29), Rn.406 ff. Sehr ausführlich hierzu Ambos, Der Allgemeine Teil des Völkerstrafrechts (2002), S. $590 \mathrm{ff}$.

78 BGHSt 40, S. 218 (236 f.); 45, S. 270 (296); dazu auch; Murmann GA 1996, S. 269 ff.; Roxin JZ 1995, S. 49 ff.; Schroeder JR 1995, S. 177 ff.; kritisch zur Annahme einer mittelbaren Täterschaft hier etwa Jakobs NStZ 1995, S. 26 f.; Rotsch NStZ 1998, S. 491 ff. 
mittelbar von der Spitze des Pentagon und des Justizministeriums ${ }^{79}$ schriftlich genehmigten Verhörmethoden, die nach oben Gesagtem als Folter oder unmenschliche Behandlung anzusehen sind ${ }^{80}$, ist die mittelbare Täterschaft derjenigen, die an der Erstellung, Genehmigung und Durchsetzung dieser Methoden nachweislich beteiligt waren, gut rekonstruierbar ${ }^{81}$.

$\mathrm{Ob}$ und wer konkret sich hier möglicherweise erfolgreich auf einen unvermeidbaren Verbotsirrtum berufen könnte, weil von höchstrangigen Regierungsjuristen Expertisen vorlagen, welche diese Methoden als zulässig betrachteten ${ }^{82}$, wäre eine Frage, die sicher nicht vor der Aufnahme von Ermittlungen sinnvoll beantwortet werden kann. Angesichts der normalerweise sehr engen Grenzen für die Annahme unvermeidbarer Verbotsirrtümer ${ }^{83}$, der Evidenz und des Gewichts der in Rede stehenden Misshandlungen $^{84}$ und der Tatsache, dass die veröffentlichten Dokumente auch eine klare, gerade auf ihre Völkerrechtswidrigkeit gestützte behördeninterne Opposition gegen diese Methoden ${ }^{85}$ belegen, wird sich aber zumindest ein großer Teil der Angezeigten hierauf letztlich nicht berufen können ${ }^{86}$. Man kann gerade diese Memoranden auch als starkes Indiz dafür auffassen, dass sich hier tatsächlich ein Machtapparat »als ganzer von den Normen des Rechts gelöst hat ${ }^{87}$.

Bezüglich der nicht unmittelbar genehmigten Methoden ist eine mittelbare Täterschaft schwieriger zu begründen. Zwar wurde der Vorwurf erhoben, die allgemeine Aufgabenstellung an das Aufsichtspersonal von Abu Ghraib, die Gefangenen für die

$79 \mathrm{Zu}$ Geheimmemos des Justizministeriums siehe Shane/Johnston/Risen in der New York Times vom 04.10.2007, unter <www.nytimes.com/2007/10/04/washington/04interrogate. html?_r=1\&hp\&oref=slogin $>$. Danach hat der Angezeigte Gonzales selbst den Einsatz eines Trommelfeuers von Schlägen, Waterboarding oder extremer Kälte (»combined effects «) gegen Gefangene der CIA zu Verhörzwecken gegen die Einwände seines damaligen Stellvertreters Comey genehmigt. Zur guten Begründbarkeit einer Organisationsherrschaft gerade für die oberste Führungsebene eines Staates siehe auch Ambos (Fn. 77), S. $603 \mathrm{f}$.

80 Es ist bezeichnend, dass Bellinger GLJ 8 (2007), S. 871 (873) hervorhebt, dass eine Order des Präsidenten vom 20.07.2007 die Anwendung des Minimalstandards des gemeinsamen Art. 3 der Genfer Konventionen auch für Geheimdienst-Gefangene verlangt. Zuvor, insbesondere in dem hier zur Anzeige gebrachten Zeitraum, galt dieser Standard augenscheinlich nicht.

81 Zur Zurechnung von Taten an höchste Verantwortungsebenen siehe Ambos (Fn. 27), § 7 Rn.32.

82 Siehe Alvarez CWRJIL 37 (2006), S. 175 (177 f.).

83 Vgl. Beck'scher Online-Kommentar StGB (Heuchemer) (BeckOK StGB) (2007), § 17 StGB, Rn.35 f.; Schönke/ Schröder (Sternberg-Lieben) (Fn. 77), § 17 StGB, Rn.14 ff.

84 Dazu Solis JICJ 2 (2004), S. 988 (995 f.).

85 Siehe etwa Bassiouni CWRJIL 37 (2006), S. 389 (396 ff.); Shane/Johnston/Risen (Fn. 79); sowie die Strafanzeige von 2006 (Fn. 6), S. 13.

$86 \mathrm{Zu}$ Gründen, trotz einer vorliegenden fehlerhaften Rechtsauskunft von einer Vermeidbarkeit des Verbotsirrtums ausgehen zu müssen, siehe Nomos Kommentar Strafgesetzbuch (Neumann) (NK), 2.Aufl. (2005), § 17 StGB, Rn. 77 ff.; Schönke/Schröder (Sternberg-Lieben) (Fn. 77), § 17 StGB, Rn.18; siehe auch Bassiouni CWRJIL 37 (2006), S. 389 (404): »fig leaf defense of the Administration's talented civilian lawyers' legal memoranda«.

87 LK (Schünemann) (Fn. 77), § 25 StGB, Rn.123. 
Verhöre »weichzukochen«, sei von vorgesetzten Offizieren ohne nähere Spezifikation der einzusetzenden Mittel erteilt worden ${ }^{88}$. Auch seien die konkret vorgefallenen Misshandlungen und Entwürdigungen von Gefangenen in diesem Zusammenhang wohlwollend von der Leitung des Gefängnisses geduldet worden ${ }^{89}$. Für diese in Abu Ghraib selbst tätigen Offiziere, die hier ebenfalls angezeigt sind, kann eine Täterschaft daher auch hierfür begründet werden ${ }^{90}$. Dies gilt umso mehr sollte sich bewahrheiten, dass die Fotos, die den Skandal losgetreten haben, nicht nur zum »Privatvergnügen « geschossen, sondern ihrerseits zur Einschüchterung weiterer Gefangener eingesetzt wurden $^{91}$, weil dann sowohl ein Interesse an der Durchführung dieser Taten seitens der Gefängnisleitung nahe liegt, als auch ihre tatsächliche Kontrolle über die ausführenden Militärpolizisten, die sich unmittelbar aus der Kommandogewalt ergibt. Damit spräche viel dafür, dass zumindest die vor Ort befindlichen höheren Ränge als Täter auch der Taten in Abu Ghraib anzusehen sind ${ }^{92}$.

Bis zu den politisch Verantwortlichen wird sich allerdings wohl nicht beweisen lassen, dass die einzelnen Taten, die über die genehmigten Methoden hinaus gingen, keine Exzesse Untergebener gegeben waren, welche von höheren Verantwortungsebenen nicht mehr beherrscht wurden. Allerdings ist hier zu prüfen, ob diejenigen, die allgemein eine Internierungs- und Befragungsstruktur befehlen, in der schwerste körperliche und seelische Misshandlungen eingesetzt werden sollen, um Informationen zu erlangen, die Entstehung eines Entschlusses zur Anwendung körperlicher und seelischer Gewalt auch über die beschriebenen Maßnahmen hinaus bei den Handelnden wenigstens billigend in Kauf nehmen ${ }^{93}$. Gleichzeitig ist es nahe liegend, dass diesen Befehlsgebern klar war, dass durch das Schaffen eines solchen Gesamtklimas und einer im Detail unspezifischen Anweisungslage die konkret handelnden Wachen dies als allgemeine Aufforderung zur Misshandlung verstehen. Auf dieser Basis können die Befehlsgeber und politisch Verantwortlichen deren Tatentschluss, über die schriftlich beschriebenen Methoden hinauszugehen, vorsätzlich verursacht, sie also im Sinne des $\S 26$ StGB angestiftet haben ${ }^{94}$.

88 Siehe etwa Solis JICJ 2(2004), S. 988 (995 f.).

89 Vgl. Taguba-Report (Fn. 14), S. 18.

90 Denn sie beherrschten zumindest den ihnen direkt unterstellten Militärapparat qua Befehlsgewalt; vgl. Ambos (Fn. 77), S. 604, der für solche unterhalb der eigentlichen Staatsspitze angesiedelten Täter wegen ihres Zusammenwirkens mit der Regierung und ihrer eigenen Abhängigkeit von ihren Vorgesetzten Mittäterschaft statt mitelbarer Täterschaft annehmen will. Auch hatten sie wohl den erforderlichen (zumindest bedingten) Vorsatz auch bezüglich der weitergehenden Misshandlungen durch ihre Untergebenen, vgl. Schönke/Schröder (Cramer/Heine) (Fn. 77), § 25 StGB, Rn.49.

91 Siehe die Meldung vom 04.08.2004 unter <www.sueddeutsche.de/ausland/artikel/541/ 36505/> sowie der Artikel »Kontroverse über die Verhömethoden der USA im Irak«, NZZ vom 14.05.2004, S. 1.

92 Vgl. Ambos (Fn. 77), S. 604.

93 Dies würde auf der subjektiven Seite für $\S 26$ StGB genügen, um nicht mehr von einem Exzess des Angestifteten zu sprechen, vgl. Schönke/Schröder (Cramer/Heine) (Fn. 77), $\S 26 \mathrm{StGB}, \mathrm{Rn} .16 \mathrm{f}$.

94 Siehe auch Ambos (Fn. 27), § 7 Rn.47; Werle (Fn. 29), Rn.411 f. 
Jedenfalls wäre es ohne die ein Klima von Gewalt, Einschüchterung und Erniedrigung beinhaltenden Anweisungen aus der politischen Führung des Pentagon zu solchen Gewaltexzessen nicht gekommen ${ }^{95}$. Hier wurden weitgehend nicht ausgebildete niedere Dienstränge mit dem Befehl versehen, die Gefangenen für Verhöre gefügig zu machen, ihnen wurde der unkontrollierte Zugriff auf ihre Opfer und die Werkzeuge für ihre Taten zur Verfügung gestellt und die Folterungen und schwersten Misshandlungen und Entwürdigungen wurden um ihres Zwecks willen zumindest hingenommen. Dies ist zumindest als psychische wie physische Beihilfe im Sinne des $§ 27$ StGB anzusehen $^{96}$.

Daneben trifft beinahe alle Vorgesetzten der Vorwurf, die Misshandlungen nicht verhindert zu haben, was bei nachgewiesenem Vorsatz den Unterlassungstatbestand des $§ 4$ VStGB erfüllt. Sollte tatsächlich für einzelne Angezeigte ein diesbezüglicher Vorsatz sich nicht erweisen, wären zumindest die $\S \S 13$ und 14 VStGB einschlägig, die auch fahrlässige Aufsichtspflichtverletzungen sowie die unterlassene unverzügliche Verfolgung von Verbrechen nach dem VStGB unter Strafe stellen ${ }^{97}$.

Insofern ist eine Zurechnung der Taten auch an die Angeklagten als militärisch oder politisch hierfür Verantwortliche selbst bei ausschließlicher Auswertung der vorliegenden Informationen möglich.

\section{b) Strafbarkeit »furchtbarer Juristen «}

Einzugehen ist allerdings noch auf eine Spezialität der hier zu besprechenden zweiten Strafanzeige. Denn anders als 2004 haben die Anzeigeerstatter ausdrücklich auch hochrangige Rechtsberater der Regierung als Tatverdächtige benannt. Diesen wird zusammengefasst vorgeworfen, in Gutachten die oben als angeordnet und genehmigt beschriebenen Foltermethoden als rechtlich zulässig und weder gegen US-Gesetze noch gegen das Völkerrecht verstoßend dargestellt zu haben. Dadurch hätten sie der politischen und militärischen Führung bewusst den Blankoscheck ausgestellt, diese Methoden systematisch einzusetzen, was als Beihilfe bewertet wird ${ }^{98}$.

Ein solcher Vorwurf kollidiert mit dem sogenannten Rechtsberatungsprivileg, wonach ein qualifizierter Rechtsberater auch dann nicht für die Umsetzung seines Rates strafrechtlich verantwortlich ist, wenn dieser sich inhaltlich als fehlerhaft erweist ${ }^{99}$. Da in der Rechtswissenschaft grundsätzlich Ansichten nur nach ihrer mehr oder weniger gegebenen Vertretbarkeit bewertet werden können, soll bei einem berufsbedingt erteilten Rechtsrat in der Regel zumindest der Vorsatz bezüglich der eigenen Unterstützung einer Haupttat fehlen ${ }^{100}$. Zumindest der äußeren Form nach wurde hier vom »Office of Legal Counsel «, einer ausschließlich für Rechtsberatung der Regierung zuständigen Abteilung des Justizministeri-

95 Alvarez CWRJIL 37 (2006), S. 175 (177 f.).

$96 \mathrm{Zu}$ deren Voraussetzungen im völkerstrafrechtlichen Kontext Ambos (Fn. 27), § 7 Rn.42 ff.

$97 \mathrm{Zu}$ diesen Normen Ambos (Fn. 27), § 7 Rn.61.

98 Strafanzeige von 2006 (Fn. 6), S. 344 ff.; Alvarez CWRJIL 37 (2006), S. 175 (176 ff.); siehe zur Strafbarkeit solchen Verhaltens nach US-Recht Bassiouni CWRJIL 37 (2006), S. 389 (398, Fn.35), der auch einen direkten Vergleich zum Nürnberger Juristenprozess zieht (S. 400 f.).

99 Siehe etwa BGH NStZ 1993, S. 43; NStZ 2000, S. 34; OLG Stuttgart NJW 1987, S. 2883.

100 So auch BGH NStZ 1993, S. 43; OLG Stuttgart NJW 1987, S. 2883. 
ums, einem entsprechenden Auftrag gemäß ein Rechtsgutachten zur Frage nach der Reichweite der durch Sec. 2340-2340A des 18. Titels des U.S. Code erstattet ${ }^{101}$. Dies könnte prima facie gegen eine Strafbarkeit der angezeigten Regierungsrechtsberater sprechen.

Die Anzeigeerstatter halten dem entgegen, dass hier bewusst nur die äußere Form eines Rechtsgutachtens eingehalten wurde und damit zu Unrecht der Anschein eines professionellen Rats erweckt werden sollte. Die inkriminierten sogenannten Foltermemos legten aber vorsätzlich eine völlig falsche Rechtslage zugrunde und kämen so zu Ergebnissen, die zwar von den Auftraggebern gewünscht, aber der Rechtsordnung sowohl der USA als auch des Völkerrechts fundamental widersprechend seien ${ }^{102}$.

Dass aber Rechtsgutachten häufig mit der Tendenz verfasst werden, möglichst gute Begründungen für ein dem jeweiligen Auftraggeber günstiges Ergebnis zu liefern, und dies auch mit der dem Ratgeber bekannten Zielrichtung, dass der Auftraggeber sich auf diesen Rat auch beruft, ist offensichtlich ${ }^{103}$. Allerdings greift die Privilegierung einer Rechtsberatung nicht mehr, wenn der Berater bewusst einen bereits tatgeneigten Haupttäter bei dessen Tat unterstützt ${ }^{104}$. Nun sind die hier zur Debatte gestellten Memoranden eine geradezu zynische Verkehrung der völkerrechtlichen und innerstaatlichen verfassungsrechtlichen Rechtslage in ihr Gegenteil ${ }^{105}$, nach der Menschen, die nur auf (häufig falschen) Verdacht hin eingesperrt werden, jeglichem Anspruch auf Achtung ihrer Rechte, ihrer Würde und ihrer Gesundheit verlustig gehen sollen. Dies haben, soweit ersichtlich, diese »furchtbaren Juristen « auch in der direkten Absicht postuliert, entgegen der ihnen bekannten tatsächlichen Rechtslage solche Methoden einzuführen und gleichzeitig den Ausführenden »Absolution« in dem Sinne zu erteilen, dass diese sich auf die Zustimmung der obersten Rechtsberater der Regierung berufen könnten ${ }^{106}$. Wenn es so etwas wie eine missbräuchliche Berufung auf ein profes-

101 Siehe »Memorandum for Alberto R. Gonzales« des Office of Legal Counsel des US-Justizministeriums vom 01.08.2002 (unter <news.findlaw.com/wp/docs/doj/bybee80102mem. pdf>), S. 1.

102 So die Strafanzeige von 2006 (Fn. 6), S. 361 ff.; Harris JNSLP 1 (2005), S. 409 (431 ff.) mit gründlicher Analyse der basalen professionellen Mängel dieser Memos; ähnlich auch Clark JNSLP 1 (2005), S. 455 (458).

103 Siehe schon RGSt 37, S. 321 (323). Allerdings weist Harris JNSLP 1 (2005), S. 409 (418) hier auf die besondere Stellung des Office of Legal Counsel hin, »to [...] provide authoritative interpretation of the law on behalf of the executive branch « (dazu näher S. $423 \mathrm{ff}$.), wodurch diese Juristen eine erheblich stärkere Verpflichtung zur Objektivität hätten, weil sie letztlich der Rechtsstaatlichkeit als solcher verantwortlich seien.

104 RGSt 37, S. 321 (323 f.); BGH NStZ 2000, S. 34; NJW 2001, S. 2409 (2410); siehe auch Wessing NJW 2003, S. 2265 (2266 f.), der dies zumindest für einen direkten Vorsatz des Beraters zugesteht, sowie Kudlich, Die Unterstützung fremder Straftaten durch berufsbedingtes Verhalten (2004), S. $458 \mathrm{ff}$.

105 Alvarez CWRJIL 37 (2006), S. 175 (178) nennt deswegen die Autorisierung von Foltermethoden durch Juristen »Torturing the Law«.

106 Strafanzeige von 2006 (Fn. 6), S. 366 ff.; Alvarez CWRJIL 37 (2006), S. 175 (177 f.); Bassiouni CWRJIL 37 (2006), S. 389 (397); siehe auch ausführlich Harris JNSLP 1 (2005), S. 409 (431 ff.). Kritisch auch das "Lawers' Statement on Bush Administration Torture Memos", ein von 106 US-amerikanischen Juristen unterzeichneter offener Brief, S. 1 f. (unter <www.hrw.org/ pub/2004/lawyers-statement.pdf >). Sehr deutlich wird dies im Begleitbrief zu dem in Fn. 101 genannten Memorandum vom Angezeigten Yoo an den Angezeigten Gonzales vom 01.08.2002, S. 5 f. (zugänglich unter <news.findlaw.com/wp/docs/doj/bybee80102ltr.html>). 
sionelles Beratungsprivileg gibt, ist dieser Missbrauch hier gegeben. Zieht man die Parallele zur Geldwäsche, wo Anwälten bei grob unprofessionellem Verhalten ebenfalls der Standesschutz entzogen worden ist ${ }^{107}$, liegt es zumindest nicht fern, hier eine Strafbarkeit wegen Beihilfe bezüglich der Umsetzung der bewusst zu Unrecht für rechtmäßig erklärten schwersten Misshandlungen anzunehmen. Die Verantwortlichkeit dieser Rechtsberater wäre jedenfalls inhaltlich zu diskutieren gewesen. Dass hier eine Verurteilung aus Rechtsgründen so unwahrscheinlich wäre, dass nicht einmal Ermittlungen zu eröffnen sein sollen, kann jedenfalls nicht angenommen werden. Die Entscheidung solcher offener Rechtsfragen wäre aber Sache der Gerichte, nicht der Staatsanwaltschaft.

Zusammenfassend ergibt die vorläufige materiellrechtliche Würdigung, dass den Angezeigten die oben genannten Verbrechen auf verschiedenen Wegen zurechenbar sein können, die Wahrscheinlichkeit einer Verurteilung von daher gegeben ist. Damit lägen aber bereits die Voraussetzungen für $§ 170$ Abs. 1 StPO vor, ein die Einleitung eines Ermittlungsverfahrens nach $\S 152$ Abs. 2 StPO erfordernder Anfangsverdacht ist hier jedenfalls gegeben.

\section{Die prozessuale Situation}

Die Generalbundesanwaltschaft hat dennoch entschieden, kein Ermittlungsverfahren einzuleiten ${ }^{108}$. Hierfür muss sich die Staatsanwaltschaft angesichts der klaren materiellen Rechtslage ausschließlich auf prozessuale Begründungen zurückziehen, wie dies auch schon bei der ersten Strafanzeige gegen Rumsfeld aus dem Jahr 2004 der Fall war $^{109}$. Um die Berechtigung der Generalbundesanwaltschaft, hier schon die Aufnahme von Ermittlungen zu verweigern, sinnvoll einordnen zu können, sollen in der Folge nach Erwägungen zu Fragen der deutschen Gerichtsbarkeit und der Immunität der Angezeigten (I.) die Voraussetzungen der hier zentralen Norm des $§ 153 f$ StPO erörtert werden (II.), bevor auch die gerichtliche Überprüfbarkeit einer solchen Entscheidung zur Nichteröffnung eines Ermittlungsverfahrens untersucht wird (III.).

\section{Verfahrensvoraussetzungen: deutsche Gerichtsbarkeit und Nichtvorliegen von Prozesshindernissen}

Die Eröffnung eines Ermittlungsverfahrens wäre unzulässig (und die Verfügung der Generalbundesanwaltschaft damit im Ergebnis zutreffend), wenn in dem angezeigten

107 BGHSt 47, S. 68 ff.; einschränkend BVerfGE 110, S. 226 ff.; siehe zur Diskussion hierüber auch Grüber/Wasserburg GA 2000, S. 430 ff. Hamm NJW 2000, S. 636 ff.; Katholnigg NJW 2001, S. 2041 ff.; Lüderssen StV 2000, S. 205 ff.; Kudlich (Fn. 104), S. 50 ff.; Schaefer/Wittig NJW 2000, S. 1387 ff.; Scherp NJW 2001, S. 3242 ff.

108 Verfügung der Generalbundesanwaltschaft vom 05.04.2007 (Fn. 7).

109 Siehe den Beschluss des OLG Stuttgart NStZ 2006, S. 117 ff., mit Besprechungen von Ambos NStZ 2006, S. 434 ff.; Basak (Fn. 5), S. 499 ff.; Singelnstein/Stolle ZIS 2006, S. 118 ff. Siehe auch die damalige Nichteröffnungsentscheidung der Generalbundesanwaltschaft, veröffentlicht in JZ 2005, S. $311 \mathrm{f}$. 
Sachverhalt keine deutsche Gerichtsbarkeit eröffnet wäre oder ein Prozesshindernis vorläge. Dies ist aber nicht der Fall.

Die Zuständigkeit deutscher Gerichte ergibt sich direkt aus $\S 1$ VStGB, der für die hier relevanten Tatbestände dieses Gesetzes uneingeschränkt das Weltrechtsprinzip postuliert und damit unabhängig von einem »nationalen Anknüpfungspunkt « den deutschen Justizbehörden die Verfolgung solcher Verbrechen gebietet ${ }^{110}$. Nach dem Willen des Gesetzgebers sollte dabei entgegen der bisherigen Praxis der Generalbundesanwaltschaft die Verfolgung solcher schwerster Verbrechen gegen Menschenrechte auch dann die Regel sein, wenn es keine unmittelbare Aussicht auf ein baldiges Strafverfahren in Deutschland geben sollte ${ }^{111}$. Es sei in diesem Zusammenhang daran erinnert, dass die Tätigkeit von Strafverfolgungsbehörden gerade im Bereich der Makrokriminalität über die reine Repression begangener Verbrechen hinaus auch Funktionen wie die öffentliche Äußerung eines Unrechtsurteils mit der Folge der Normbestätigung und -implementierung oder der öffentlichen Solidaritätsbekundung mit den Opfern und der Delegitimierung der Täter hat ${ }^{12}$. Zudem besteht auch im Hinblick auf möglicherweise erst in fernerer Zukunft zu führende Prozesse ein eminentes Interesse daran, Beweise justiziell zu sichern und damit zugänglich zu halten, so lange diese noch greifbar sind ${ }^{113}$. Verwiesen sei hier vor allem auf schriftlich in Justizakten zu dokumentierende Zeugenaussagen, die mit zunehmendem Zeitabstand deutlich an Qualität verlieren. Es ist Intention des VStGB und seines Gesetzgebers, dass die deutschen Ermittlungsbehörden ihre Arbeit so weit treiben, wie ihnen dies möglich ist ${ }^{114}$. Entsprechend ist die weit ausgestaltete Zuständigkeit der deutschen Justiz hier eröffnet und intendiert.

Einer Strafverfolgung durch die deutsche Justiz steht auch für keinen der hier Angezeigten ein Strafverfolgungshindernis im Wege. In Betracht käme hier nur eine völkerrechtlich gegebene Immunität nach $\S 20 \mathrm{GVG}^{115}$.

Maßgeblich für die Frage nach einer Immunität ist, ob der jeweilige Amtsträger amtlich oder nichtamtlich handelt und ob er sich noch im Amt befindet oder nicht mehr ${ }^{116}$. So kommt personelle (absolute) Immunität, die unabhängig von der Natur der jeweiligen Handlung - ob dienstlich oder privat - vor allen Zugriffen der Gerichtsbarkeit fremder

110 BR-DruckS. 29/02, S. 29; Ambos NStZ 2006, S. 434 f.; Löwe-Rosenberg, StPO (LR) (Beulke) 25.Aufl., Nachtrag (2003), § 153c StPO, Rn.1; Basak (Fn. 5), S. 499 (501); Fischer-Lescano KJ 2005, S. 72 (75); Satzger NStZ 2002, S. 125 (131 f.); siehe auch Werle (Fn. 29), Rn.173.

111 BT-DruckS. 14/8892, S. 38; vgl. auch Kreß NStZ 2000, S. 617 (624 f.)

112 Zappalà JICJ 4 (2006), S. 602 (606); siehe auch Neubacher in; Neubacher/Klein, Vom Recht der Macht zur Macht des Rechts? (2006), S. 17 (29 ff.).

113 Zur Wichtigkeit der Dokumentation historischer Fakten bezüglich Makroverbrechen durch Strafprozesseauch Steinbach in Neubacher/Klein (Fn. 112), S. 139 ff.

114 BT-DruckS. 14/8892, S. 38; Systematischer Kommentar zur StPO (SK-StPO) (Weßlau), 32.Lfg. (2003), § 153f StPO, Rn.13; Basak (Fn. 5), S. 499 (513 f.).

115 Zur Immunität als Verfahrenshindernis siehe KK (Schoreit) (Fn. 76), § 153f StPO, Rn.3; Ambos (Fn. 27), § 7 Rn.106.

116 Graf Vitzthum (Hailbronner), Völkerrecht, 4. Aufl. (2007), S. 168; Ipsen, Völkerrecht, 5. Aufl. (2004), S. 377. 
Staaten schützt, außerhalb des spezielleren Diplomatenrechts (WÜD) jedenfalls Staatsoberhäuptern $\mathrm{zu}^{117}$. Weil es dabei um eine Ausprägung der Staatenimmunität und mithin der souveränen Gleichheit der Staaten geht, besteht sie absolut nur während der Amtszeit. Hingegen ist ein nicht mehr amtierendes Staatsoberhaupt nur noch im Rahmen funktioneller Immunität, das heißt wegen dienstlicher Handlungen in Ausübung der amtlichen Stellung, vor der Gerichtsbarkeit anderer Staaten geschützt. Dies rechtfertigt sich, weil bei amtlichem Handeln a priori keine individuelle strafrechtliche Verantwortlichkeit entsteht, sondern eine Zurechnung der Handlung zum Staat selbst stattfindet ${ }^{118}$. Äußerst fraglich ist aber, wie weit anderen Regierungsmitgliedern personelle Immunität zukommt. Abgesehen von Staatsoberhäuptern sind jedenfalls für Regierungschefs und Außenminister, die in der Staatenpraxis den Staat nach außen repräsentieren, diese Immunitätsgrundsätze anwendbar ${ }^{119}$. Dies lässt sich aber nicht auf Verteidigungs- oder Justizminister übertragen, weil diese nicht die primäre Funktion haben, ihren Staat nach außen zu repräsentieren ${ }^{120}$. Auch ist eine solche weitergehende Ausdehnung der Immunität unnötig, da Minister bei dienstlichen Aufenthalten im Ausland entsprechend der Regeln über diplomatische Spezialmissionen wie Diplomaten zu behandeln ${ }^{121}$ und damit dem Zugriff fremder Strafgerichtsbarkeit ohnehin entzogen sind. Dem dient eben $§ 20$ Abs. 1 GVG.

Bezogen auf die hier erhobenen Vorwürfe stellt sich allerdings die Frage, ob die Verteidigungsminister Rumsfeld vorgeworfene Anordnung von Folterhandlungen begrifflich als »nichtamtlich« eingeordnet werden kann, oder ob zwar die amtliche Natur von Folterhandlungen zu bejahen ist, sich insoweit aber eine völkergewohnheitsrechtliche Ausnahme von der Immunität ratione materiae herausgebildet hat, die ein Verfahrenshindernis darstellen würde. Denn stuft man die vorliegenden Vorwürfe gegen Rumsfeld und andere Staatsbedienstete als amtlich ein und lässt sich keine völkergewohnheitsrechtliche Regel über den Ausschluss der Immunität bei völkerrechtlichen Verbrechen feststellen, wäre ein Verfahren in Deutschland gegen sie nicht möglich ${ }^{122}$.

Dass es sich bei Folter schon gar nicht um amtliche Handlungen, sondern um solche bereits begrifflich ausschließende »international crimes « handle, vertrat das britische House of Lords in seiner ersten Pinochet- Entscheidung ${ }^{123}$ und verneinte in der Konsequenz eine

117 Vgl. BGHSt 33, 97 (Fall Honnecker); Murphy AJIL 2003, S. 962 (974 ff.).

118 Vgl. Graf Vitzthum (Hailbronner) (Fn. 116), III. Abschn., Rn.46 ff.; Strafanzeige von 2006 (Fn. 6), S.373 f. Zum fiktionalen Charakter des völkerrechtlichen Schutzes, der bei einer individuellen Rechtsverletzung auch die Betroffenheit des Heimatstaates des Opfers und damit eine Staatenverantwortlichkeit fingiert, siehe auch Vermeer-Künzli EJIL 18 (2007), S. $37 \mathrm{ff}$.

119 IGH Kongo ./. Belgien ILM 41 (2002), S. 536 ff.; insoweit zustimmend Cassese EJIL 13 (2002), S. 853 (855).

120 Hierzu Wirth EJIL 13 (2002), S. 877 (879).

121 Ipsen (Fn. 116), S.384, siehe auch Schweisfurth, Völkerrecht (2006), S. 113; Zappalà JICJ 4 (2006), S. 602 (616).

122 Dies würde auch nicht nur Rumsfeld als früheren Minister, sondern alle Verdächtigen betreffen, deren Handlungen völkerrechtlich dem Staat als dessen Akte zugerechnet werden könnten, also auch alle Militärs und Geheimdienstmitarbeiter, vgl. Wirth EJIL 13 (2002), S. 877 (881 ff.).

123 Entscheidung des House of Lords vom 25.11.1998, zugänglich unter <www.publications. parliament.uk/pa/ld199899/ldjudgmt/jd981125/pino01.htm>. 
funktionelle Immuntität. Ähnlich argumentiertern die Richter Higgins, Kooijmans und Buergenthal in ihrem Sondervotum zum Fall Kongo gegen Belgien, wonach sich kein Amtsträger nach Ende der Amtszeit auf Immunität berufen könnte, um einer Strafverfolgung wegen schwerster Menschenrechtsverletzungen zu entgehen ${ }^{124}$. Weil der offizielle Status und die mit dem Amt verbundenen Befugnisse in der Regel erst die Begehung völkerstrafrechtlicher Tatbestände ermöglichen ${ }^{125}$, erscheint die Einordnung dieser Handlungen als nichtamtlich aber nicht konsequent. Gerade Art. 1 Abs. 1 CAT setzt für den Begriff der Folter definitorisch bereits amtliches Handeln voraus, weshalb es verfehlt erscheint, Folter als »privat« einzustufen. Hierfür spricht auch, dass sich Staaten die Verletzungen völkerrechtlicher Pflichten auch dann zurechnen lassen müssen, wenn das handelnde Organ ultra vires gehandelt hat, solange die Handlung für den Staat erfolgte ${ }^{126}$. Wird Folter dennoch als »privat« eingestuft, wird eine Zurechnung und damit die völkerrechtliche Verantwortlichkeit des Staates, dessen Amtsträger gefoltert hat, verhindert: Private Handlungen seiner Amtsträger muss sich ein Staat nach allgemeiner Ansicht nicht zurechnen lassen ${ }^{127}$. Umgekehrt verhindert aber die persönliche Haftbarkeit eines Regierungsmitgliedes oder Staatsbediensteten gerade nicht die Staatenhaftung für dessen Taten ${ }^{128}$.

Damit ist hier nach völkergewohnheitsrechtlichen Ausschlussgründen der Immunität zu fragen ${ }^{129}$. Da sich funktionale Immunität nach Völkerrecht und Völkerstrafrecht, dessen Begehung schon von der Natur seiner Tatbestände her staatsverstärktes Handeln ${ }^{130}$ voraussetzt, in einem offenen Spannungsverhältnis befinden, ist nach dessen Auflösung $\mathrm{zu}$ fragen ${ }^{131}$. Das oft zitierte Urteil des IGH im Fall Kongo gegen Belgien $^{132}$ tritt scheinbar den Tendenzen, eine Strafverfolgung ausländischer Regierungsmitglieder zuzulassen, entgegen ${ }^{133}$ Zwar betraf der Fall die Immunität eines Außenministers während der Amtszeit, die vom IGH - zu Recht - in oben dargestelltem Umfang bejaht wurde. In einem obiter dictum wurde aber die Möglichkeit einer Strafverfolgung durch einen Drittstaat für einen Außenminister nur für während seiner Amtszeit verübte »private«, also nichtamtliche, Handlungen bejaht ${ }^{134}$.

124 IGH Kongo ./. Belgien, Gemeinsames Sondervotum der Richter Higgins, Kooijmans and Buergenthal, ILM 41 (2002), S. 575 (591, Ziff.85).

125 Ambos (Fn. 27), § 7 Rn.112; Kreß NStZ 2000, S. 617 (620 f.).

126 Vgl. Art. 7 Entwurfs der International Law Commission zur Staatenverantwortlichkeit, Report of the ILC, GA- Records $56^{\text {th }}$ session, Suppl. No.10 (A/56/10), S. 43 ff.; siehe auch Zappalà JICJ 4 (2006), S. 602 (614).

127 So Spinendi EJIL 2002, S. 895 (897 ff.).

128 Cassese EJIL 13 (2002), S. 853 (864); siehe hierzu näher auch Mohr in: Hankel/ Stuby (Hrsg.), Strafgerichte gegen Menschheitsverbrechen (1995), S. $401 \mathrm{ff}$.

129 So auch jüngst das House of Lords in Jones v Ministry of Interior Al Mamlaka Al Arabiya Al Saudiya and others vom 14.06.2006, Ziff.19 und 84 (unter <www.publications.parlia ment.uk/pa/ld200506/ldjudgmt/jd060614/jones-1.htm>.

130 Zum Begriff Naucke, Die strafjuristische Privilegierung staatsverstärkter Kriminalität (1996).

131 Kreß NStZ 2000, S. 617 (620 f.).

132 IGH Kongo ./. Belgien ILM 41 (2002), S. 536 ff.

133 Herdegen Völkerrecht, 6.Aufl. (2007), S. 247.

134 IGH Kongo ./. Belgien ILM 41 (2002), S. 536 (552, Ziff.61): »Provided that it has jurisdiction under international law, a court of one State may try a former minister of foreign affairs of another state in respect of acts committed prior or subsequent to his or her period of office, as well as in respect of acts committed during that period of office in a private cappacity.«. 
Diese restriktive Auslegung der Immunitätsregeln wurde intensiv kritisiert ${ }^{135}$. Allerdings ist nicht klar, ob dieser Ausspruch Ausschließlichkeit beanspruchen will oder nur als Beispiel gelten soll, das auch weitere Ausnahmen von der funktionellen Immunität zulässt $^{136}$. Dafür spricht auch, dass die dem völkergewohnheitsrechtlich hier anerkannten Weltrechtsprinzip ${ }^{137}$ unterfallenden völkerstrafrechtlichen Tatbestände zwar nicht denknotwendig, aber doch regelmäßig durch zumindest staatlich unterstütztes und damit amtliches Handeln im Sinne des Völkerrechts verübt werden ${ }^{138}$. Die Anerkennung einer funktionellen Immunität für die Begehung solcher Verbrechen ließe das Weltrechtsprinzip völlig ins Leere laufen und widerspräche auch den einzelstaatlichen Verfolgungsund Bestrafungspflichten ${ }^{139}$. Dies deckt sich mit der allgemeinen Entwicklung, völkerrechtliche Verbrechen als Verstoß gegen den ius cogens als Verletzung der Interessen aller Staaten und nicht als innere Angelegenheit anzusehen ${ }^{140}$. Hierfür spricht auch die völkerrechtliche Rechtsentwicklung, etwa die 6. Präambelerwägung des ICC-Statuts, die eine funktionelle Immunität für solche Verbrechen klar verneint ${ }^{141}$. Insofern ist die häufig anzutreffende Feststellung, eine Immunität ratione materiae sei bei völkerstrafrechtlichen Verfahren auch vor nationalen Gerichten in Drittstaaten völkergewohnheitsrechtlich ausgeschlossen ${ }^{142}$, im Ergebnis zutreffend.

135 IGH Kongo ./. Belgien, Gemeinsames Sondervotum der Richter Higgins, Kooijmans and Buergenthal, ILM 41 (2002), S. 575 (591, Ziff.85); kritisch auch etwa Schultz ZaöRV 62 (2002), S. 703 ff.; Wirth EJIL 13 (2002), S. 877 ff.; Zeichen/Hebenstreit AVR 41 (2003), S. 182 ff.

136 So etwa IGH, Presseerklärung von Richter Gilbert Guillaume vom 14.02.2002 (»by way of example«); siehe hierzu auch Cassese EJIL 13 (2002), S. 853 (876); Werle (Fn. 29), Rn.455, Fn.416.

137 Siehe hierzu SCSL, Entscheidung der Appeals Chamber im Fall Kallon und Kamaro über die im Lomé-Abkommen enthaltene Amnestie vom 13.03.2004, Ziff.69 ff.; Ambos (Fn. 27), § 3 Rn.96; Bothe/Fischer-Lescano, Die Bedeutung völkerrechtlicher Bestrafungspflichten und der völkergewohnheitsrechtlichen Jurisdiktions- und Immunitätsregeln für Verfahren nach dem Völkerstrafgesetzbuch (2005), S. 2 ff. (zugänglich unter <www.rav.de /downloads/RumsfeldKurzgutachten1.pdf>); Cassese EJIL 13 (2002), S. 853 (859 ff.); Hessbrügge (Fn. 2), 1 A; Kreicker in: Eser/Kreicker (Hrsg.), Nationale Strafverfolgung völkerrechtlicher Verbrechen. Bd.1: Deutschland (2003), S. 253; Kreß, Vom Nutzen eines deutschen Völkerstrafgesetzbuches (2000), S. 26 und JICJ 4 (2006), S. 561 (569 ff.); Kurth ZIS 2006, S. 81 (82); Satzger NStZ 2002, S. 125 (131 f.); Weigend in: Festschrift für Eser (2005), S. 955 (962 ff.); Werle/Jeßberger JZ 2002, S. 725 (729).

138 Ambos (Fn. 27), § 7 Rn.112; Kreß NStZ 2000, S. 617 (620 f.).

139 Vgl. Kreß NStZ 2000, S.617 (621), sowie in der Einlassung zur Anhörung des Menschenrechtsausschusses des Bundestages vom 24.10.2007, S. 9 f. (unter <www.bundestag.de/aus schuesse/a17/anhoerungen/voelkerstrafgerichtshof/Stellungnahme_Kress.pdf>; zu letzteren Bothe/Fischer-Lescano (Fn. 137), S. 4 ff.; Schmidt, Externe Strafpflichten (2002), S. $131 \mathrm{ff}$.

140 Ambos (Fn. 27), § 7 Rn.111; Zappalà JICJ 4 (2006), S. 602 (617). Zur Verletzung des ius cogens durch Kriegsverbrechen und Verbrechen gegen die Menschlichkeit siehe auch die Entscheidung des italienischen Kassationsgerichts im Fall Ferrini, Rivista di diritto internatzionale 87 (2004), S. 539 ff.; dazu auch De Sena/De Vittor EJIL 16 (2005), S. 89 ff.; Gattini JICJ 3 (2005), S. 224 ff.

141 Siehe hierzu auch Garraway JICJ 2 (2004), S. 981 (982).

142 ICTY, Urteil der Appeals Chamber im Fall Blaškić vom 29.10.1997, Ziff.41; Bothe/ Fischer-Lescano (Fn. 137), S. 12; Cassese EJIL 13 (2002), S. 853 (870); De Sena/De Vittor EJIL 16 (2005), S. 89 (104); Herdegen (Fn. 133), S. 253; Kreß GA 2003, S. 25 (35); Werle (Fn. 29), Rn.455; Zappalà JICJ 4 (2006), S. 602 (613 f.). 
Damit scheidet eine allgemeine funktionelle Immunität für Verbrechen gegen das Völkerstrafrecht auS. Auf eine diplomatische Immunität können sich Justiz- oder Verteidigungsminister (weder im Amt befindliche noch wie hier zurückgetretene) zunächst ebenso wenig berufen wie Offiziere der Armee oder ehemalige Geheimdienstmitarbeiter. In Frage käme höchstens die Figur einer ad hoc entstehenden diplomatischen Immunität, etwa für Spezialmissionen ${ }^{143}$. Dass eine solche aber im Einzelfall entstehen könnte und in bestimmten Konstellationen, wie etwa der Teilnahme an Regierungsdelegationen oder der Teilnahme an von der Bundesregierung veranstalteten Tagungen und Konferenzen gemäß $§ 20$ GVG gegeben wäre, spielt für die Frage nach einem aktuell vorliegenden Prozesshindernis keine Rolle. Die Frage, ob ein wegen der Beteiligung an völkerstrafrechtlichen Verbrechen Verdächtigter im Einzelfall tatsächlich von seinem Heimatstaat unter den Schutz dieser Immunität genommen wird, und ob dies auch vom Bundesaußenministerium bestätigt wird ${ }^{144}$, ist eine politische, deren Antizipation der Generalbundesanwaltschaft weder möglich ist noch zusteht. Aktuell besteht ein solches Prozesshindernis nicht, deswegen kann die Nichteröffnung eines Ermittlungsverfahrens hierauf auch nicht gestützt werden.

\section{Die Voraussetzungen des $\S 153 f$ StPO}

Die Nichteröffnung des Verfahrens kann damit nur noch auf $\S 153 f$ StPO gestützt werden, auf den sich die Generalbundesanwaltschaft auch beruft. Diese Norm, die eigentlich eine Ermächtigung zur Verfahrenseinstellung darstellt, soll auch eine Nichtverfolgungsermächtigung beinhalten, weil es sinnlos sei, ein Verfahren zu eröffnen, um es dann unmittelbar wieder über diese Norm einzustellen ${ }^{145}$. Diese Erwägung erscheint plausibel, allerdings muss vor einer genaueren Untersuchung des Vorliegens der Voraussetzungen der Norm eines festgehalten werden: Auch wenn $§ 153 f$ StPO über seinen Wortlaut hinaus auch als Ermächtigung zur Nichteröffnung eines Ermittlungsverfahrens eingesetzt wird, ändert dies nichts an dem Inhalt der Norm. Anders ausgedrückt: Die Tatbestandsmerkmale der Norm, die eine Einstellung erst erlauben ${ }^{146}$, müssen auch bei der Entscheidung über eine Nichtaufnahme von Ermittlungen in vollem Umfang vorliegen und dürfen nicht etwa mit dem Hinweis auf derzeit nicht vorliegende Erkenntnisse abgeschwächt werden. Wenn diese Tatbestandsmerkmale nicht positiv begründet werden können, bedeutet dies eben, dass die Generalbundesanwaltschaft zunächst in Ermittlungen eintreten und in deren Rahmen das Vorliegen der Merkmale des $\S 153 f$ StPO klären musS. Hieran wird sich die folgende Betrachtung orientieren. Diese wird sich zunächst auf die Frage nach der tatsächlichen und auch künftig zu erwartenden Abwesenheit der Angezeigten im Bundesgebiet nach $\S 153 \mathrm{f}$ Abs. 1 StPO konzentrieren (1.) und dann die Frage einer Strafverfolgung in den USA diskutieren (2.), bevor auf den Umfang eines möglicherweise eröffneten Ermessens eingegangen wird (3.).

143 Siehe dazu BGH NJW 1984, S. 2048 ff. (Fall Tabatabai).

144 So der Ablauf im Fall Tabatabai, siehe BGH NJW 1984, S. 2048 (2049).

145 LR (Beulke) (Fn. 110), § 153f StPO, Rn.14.

146 LR (Beulke) (Fn. 110), § 153f StPO, Rn.7. 


\section{Die Zulässigkeit einer Ermittlungsverweigerung nach $\S 153 \mathrm{f}$ Abs. $1 \mathrm{StPO}$}

$\S 153 \mathrm{f}$ StPO wird als »prozessrechtliche Flankierung « oder auch als prozessuale Einschränkung des in $\S 1$ VStGB postulierten Weltrechtsprinzips angesehen, die eine Überforderung der deutschen Justizbehörden vermeiden soll, welche bei strenger Geltung des Legalitätsprinzips in diesem Bereich befürchtet wurde ${ }^{147}$. Praktisch ist zu konstatieren, dass von einer solchen Überforderung bislang keine Rede sein kann, hat doch die Generalbundesanwaltschaft in praktisch jedem bei ihr angezeigten Fall schon die Aufnahme von Ermittlungen verweigert ${ }^{148}$.

Andererseits spricht aus § 153f StPO eindeutig der Wille, das Legalitätsprinzip für den Bereich der Völkerrechtsverbrechen gegenüber der für sonstige Auslandsstrafverfolgungen geltenden Norm des $\S 153 \mathrm{c}$ StPO deutlich zu stärken ${ }^{149}$. Anders als die letztgenannte Norm eröffnet §153f StPO die Möglichkeit der Einstellung (und damit auch der Ermittlungsverweigerung) nur bei Vorliegen bestimmter Voraussetzungen, zu denen explizit nicht die außenpolitischen Interessen der Bundesrepublik gehören ${ }^{150}$. Dies war eine bewusste Entscheidung des Gesetzgebers, da es bei solchen Verbrechen keine akzeptablen außenpolitischen Gründe für eine Straflosigkeit der Täter geben soll.

Nach $\S 153 \mathrm{f}$ Abs. 1 StPO ist eine Verfahrenseinstellung nur dann zulässig, wenn sich kein Tatverdächtiger im Inland aufhält und ein solcher Aufenthalt auch in Zukunft nicht zu erwarten is ${ }^{151}$. Dabei ist der Begriff »Aufenthalt« sehr weit zu verstehen und umfasst praktisch jedes Betreten des Bundesgebietes, selbst den Transit auf einer Durchreise oder gar eine Notlandung im Bundesgebiet ${ }^{152}$. Eine Strafverfolgung soll immer dann Pflicht der Staatsanwaltschaft bleiben, wenn physisch eine Verhaftung durch deutsche Behörden zumindest möglich ist ${ }^{153}$. So wird ein relativ weiter Bereich der Strafverfolgung vom Gesetzgeber bewusst Opportunitätserwägungen ganz entzogen.

Die dahinter stehende Grundhaltung einer weitgehenden Verfolgungspflicht in allen Fällen, in denen ein Verfahren möglich ist ${ }^{154}$, strahlt auch auf das Verständnis der Wendung, dass ein Aufenthalt im Inland nur »zu erwarten« sein muss, um eine Verfahrenseinstellung auszuschließen. Legt man dies parallel zu dem Begriff des Aufenthaltes weit aus, so muss auch hier die Möglichkeit eines solchen Aufenthaltes genü-

147 Ambos (Fn. 27), § 3 Rn.100; Meyer-Goßner (Fn. 76), § 153f StPO, Rn.1; SK-StPO (Weßlau) (Fn. 114), § 153f StPO, Rn.1.

148 Siehe auch Ambos (Fn. 27), § 3 Rn.103.

149 KMR (Plöd) (Fn. 5), § 153f StPO, Rn.4; SK-StPO (Weßlau) (Fn. 114), § 153f StPO, Rn.2; Pfeiffer, Strafprozessordnung, 5.Aufl. (2007), § 153f StPO, Rn.1; Joecks, Strafprozessordnung, Studienkommentar (2006), § 153f StPO, Rn.1.

150 LR (Beulke) (Fn. 110), § 153c StPO, Rn. 3; Kreß (Fn. 139), S. 5 f.

151 KMR (Plöd) (Fn. 5), § 153f StPO, Rn.4; Joecks (Fn. 149), § 153f StPO, Rn.1.

152 BT-DruckS. 14/8524, S. 38; KMR (Plöd) (Fn. 5), § 153f StPO, Rn.8; SK-StPO (Weßlau) (Fn. 114), § 153f StPO, Rn.9.

153 SK-StPO (Weßlau) (Fn. 114), § 153f StPO, Rn.9.

154 Vgl. auch KMR (Plöd) (Fn. 5), § 153f StPO, Rn.4; LR (Beulke) (Fn. 110), § 153f StPO, Rn.4 weist auf das vorrangige Ziel auch des $§ 153$ f StPO hin, eine Straflosigkeit der Täter völkerrechtlicher Verbrechen zu verhindern. 
gen ${ }^{155}$. Dies ergibt sich auch aus den in $\S 153 \mathrm{f}$ StPO verteilten Begründungslasten. $\S 153 \mathrm{f}$ Abs. 1 S. 1 und Abs. 2 S. 1 Nr. 3 StPO verwenden den Begriff in Negation »nicht zu erwarten « - als Voraussetzung für die Eröffnung eines Einstellungsermessens der Staatsanwaltschaft. Danach obliegt es nach allgemeinen prozessrechtlichen Grundsätzen der Staatsanwaltschaft, wenn Sie sich hierauf berufen will, plausibel und faktengestützt zu belegen, warum ein Aufenthalt der Verdächtigen im oben beschriebenen weiten Sinne auch in Zukunft »nicht zu erwarten « ist ${ }^{156}$. Kann dies nicht substantiiert begründet werden, greift die Ausnahmeregelung des $§ 153 \mathrm{f}$ StPO nicht und es gilt das allgemeine Legalitätsprinzip des $§ 152$ StPO. Der reine Verweis darauf, dass nichts dafür spricht, dass die betreffende Person demnächst einreisen könnte, reicht hier eben nicht. Es ist nicht Sache der Anzeigenden, die Erwartung für einen künftigen Aufenthalt zu belegen. Vielmehr muss die Staatsanwaltschaft für eine Entscheidung zur Verfahrenseinstellung nach $§ 153 \mathrm{f}$ StPO ihrerseits Fakten beibringen, welche die Möglichkeit einer solchen künftigen Einreise fernliegend erscheinen las$\operatorname{sen}^{157}$. Nur so kann der vorrangige Zweck auch des $§ 153 \mathrm{f}$ StPO, nämlich die Verhinderung von Straflosigkeit für Täter völkerrechtlicher Verbrechen ${ }^{158}$, gesichert werden. Wenn sich die Staatsanwaltschaft hingegen darauf beruft, dieser bestünde in der Vermeidung »fruchtloser Ermittlungsarbeit ${ }^{159}$, hier bezüglich des aktuellen und künftigen Aufenthaltsstatus von Verdächtigen, so verkennt sie einerseits die Rangfolge der Gesetzeszwecke und andererseits die Systematik der Norm ${ }^{160}$.

Wie gesagt betrifft dies den von der Norm intendierten Regelfall einer Einstellung eines laufenden Ermittlungsverfahren, in dem die Staatsanwaltschaft eine solche Begründung auf der Basis ihrer gewonnenen Erkenntnisse durchaus schlüssig erbringen könnte. Wird aber $\S 153 \mathrm{f}$ StPO wie vorliegend erweiternd als Ermächtigung gebraucht, gar keine Ermittlungen aufzunehmen, können diese Voraussetzungen für die Anwendung der Norm nicht abgesenkt werden. Regelmäßig wird die Frage, ob ein Inlandsaufenthalt nicht vorliegt oder auch künftig nicht zu erwarten ist, ohne die Aufnahme von Ermittlungen - eben zum Aufenthaltsort der Verdächtigen sowie zu deren persönlichen Umständen - gar nicht zu beantworten sein. Eine Nichtaufnahme von Er-

155 So auch zu Recht der Klageerzwingungsantrag (Fn. 8), unter 4.1.1.3.

156 Siehe auch Zappalà JICJ 4 (2006), S. 602 (607 f.). Parallel hierzu sieht auch das spanische Verfassungsgericht (Urteil 237/2005 vom 26.09.2005, unter II.4., <www.tribunalconstitu cional.es/jurisprudencia/JC2005.html>) die Voraussetzungen für die Ausübung universeller Jurisdiktion zumindest teilweise auch als Problem der Beweislast, die so verteilt sein müsse, dass das Ziel der Verhinderung von Straflosigkeit schwerster Menschenrechtsverbrechen erreicht werden könne, dazu Ascensio JICJ 4 (2006), S. 586 (590 f.).

157 Siehe auch LR (Beulke) (Fn. 110), § 153f StPO, Rn.16, der bei grundsätzlich gleichem Ansatz hier etwas großzügiger sein will, aber selbst »Wertungswidersprüche« zu dem Ziel eines Ausschlusses von Straflosigkeit sieht. Diese lassen sich aber durch die ausgeführte Begründungslast auf Seiten der Staatsanwaltschaft befriedigend ausräumen.

158 LR (Beulke) (Fn. 110), § 153f StPO, Rn.4. Genau diese Straflosigkeit ist aber nach durchaus berechtigter Ansicht der Anzeigeerstatter im vorliegenden Fall zu befürchten, siehe Klageerzwingungsantrag (Fn. 8), unter 2.2.

159 Verfügung der Generalbundesanwaltschaft vom 05.04.2007 (Fn. 7), S. 6 unter Berufung auf LR (Beulke) (Fn. 110), § 153f StPO, Rn.5.

160 Siehe auch Klageerzwingungsantrag (Fn. 8), unter 4.1.2.2. 
mittlungen käme demnach überhaupt nur dann in Betracht, wenn evident ist, dass jeder Bezug zum Bundesgebiet fehlt und auch dauerhaft fehlen wird. Bei einem somalischen Warlord mag dies etwa vorstellbar sein. Besteht oder bestand aber eine berufliche oder persönliche Verbindung der Verdächtigen zur Bundesrepublik oder haben diese sich gar nachweislich bereits im Bundesgebiet aufgehalten, ist eine solche Evidenz nicht gegeben ${ }^{161}$. Dann ist schon durch die genannte Begründungslast eine Berufung auf $\S 153 \mathrm{f} \mathrm{StPO}$ als Ermächtigung zur Nichtermittlung praktisch ausgeschlossen.

Demgegenüber beruft sich die Generalbundesanwaltschaft in ihrer Nichteröffnungsverfügung lapidar darauf, dass sich hier kein Verdächtiger im Inland aufhalte und dies auch künftig nicht zu erwarten sei. Damit sei eine Ablehnung der Ermittlungen nach § 153f Abs. 1 StPO möglich ${ }^{162}$. Belegt wird diese weitreichende Behauptung lediglich durch einen Verweis auf eine informell eingeholte Auskunft eines Rechtsberaters der US-Streitkräfte in Deutschland, welcher die Staatsanwaltschaft offenbar ohne weiteres Glauben schenken will ${ }^{163}$. Dies wird aber den soeben entwickelten Anforderungen an eine substantiierte Begründung der Voraussetzungen des $§ 153 \mathrm{f}$ Abs. 1 StPO nicht einmal ansatzweise gerecht ${ }^{164}$. Vielmehr scheint die Generalbundesanwaltschaft die Begründungslast für eine positive Einreiseprognose bei den Anzeigeerstattern zu sehen ${ }^{165}$. Dies verkennt aber, wie gesagt, den Ausnahmecharakter des § 153f StPO und den Wortlaut der Norm.

Zudem ist hier anzumerken, dass sich eine Auskunft aus den Reihen der Streitkräfte nur auf Armeeangehörige beziehen kann, nicht aber auf hier ebenfalls angezeigte Zivilisten. Für diese fehlen also alle Hinweise auf einen Grund für die Annahme einer auch künftig nicht zu erwartenden Einreise.

Bei den Militärs ist aber schon angesichts des zentralen Charakters der in der Bundesrepublik liegenden US-Basen, die als primärer Umschlagplatz für Material und Personal auf dem Weg in den Nahen und Mittleren Osten dienen, jedenfalls ohne sehr stichhaltige Gegenargumente eine künftige Einreise im Sinne des § 153f StPO als hoch wahrscheinlich anzusehen ${ }^{166}$. Hierfür spricht auch, dass zumindest bei der ersten

161 Wenn SK-StPO (Weßlau) (Fn. 114), § 153f StPO, Rn.9 für Prognose eines künftigen Aufenthaltes eines Verdächtigen die Feststellung ausreichen lassen will, dass »irgendwelche beruflichen, persönlichen oder familiären Bindungen in Deutschland « bestehen (zitiert auch von der Verfügung der Generalbundesanwaltschaft vom 05.04.2007 [Fn. 7], S.5), heißt dies auch, dass bei Hinweisen auf solche Beziehungen eine Einstellung ausscheidet. Zappalà JICJ 4 (2006), S. 602 (607 f.) will gar bei einem nach der Tat einmal gegebenen Aufenthalt eines Verdächtigen in Deutschland § 153f StPO ganz ausschließen, weil damit unabhängig von einer Strafanzeige schon von Amts wegen eine Verfolgungspflicht der Staatsanwaltschaft konstitutiert sei, der sie sich nicht durch reine Untätigkeit entziehen könne, ohne sich dem Vorwurf des Missbrauchs ihrer Befugnisse auszusetzen.

162 Verfügung der Generalbundesanwaltschaft vom 05.04.2007 (Fn. 7), S. 5.

163 Verfügung der Generalbundesanwaltschaft vom 05.04.2007 (Fn. 7), S. 5; siehe auch den Klageerzwingungsantrag (Fn. 8), unter 2.3, der eine weitere Verfügung der Generalbundesanwaltschaft vom 11.07.2007 entsprechend zitiert, und unter 4.1.1.1 mit Hinweis auf den Akteninhalt.

164 Vgl. auch LR (Beulke) (Fn. 110), § 153f StPO, Rn.16.

165 Verfügung der Generalbundesanwaltschaft vom 05.04.2007 (Fn. 7), S. 6.

166 Siehe zu konkreteren Angaben bezüglich einzelner angezeigter Militärs die Strafanzeige von 2006 (Fn. 6), S. 82. 
Anzeige aus dem Jahr 2004 der überwiegende Anteil der damals wie heute angezeigten Offiziere in Deutschland stationiert waren ${ }^{167}$. Diese Personen hatten also zumindest zeitweise einen Wohnsitz im Bundesgebiet. Zudem besteht eine sogar recht starke berufliche Verbindung zum Bundesgebiet. Um hier die Möglichkeit einer künftigen Einreise mit hinreichend großer Wahrscheinlichkeit ausschließen zu können, müsste die Generalbundesanwaltschaft intensive Ermittlungen dazu anstellen, was jede einzelne dieser Personen im Moment genau beruflich und privat für Kontakte nach Deutschland hat, ob sich die Einheiten der Offiziere in Deutschland befinden, warum für gerade diese Personen ein künftiger Einsatz etwa im Irak, bei dem sie auf dem Weg mit sehr hoher Wahrscheinlichkeit mit dem Bundesgebiet in Berührung kämen, ausgeschlossen sein soll und so fort ${ }^{168}$. Mit einem Anruf im Hauptquartier der US-Streikräfte in Deutschland ist dies nicht zu erledigen. Allein um dies begründen zu können, müssten erst einmal Ermittlungen aufgenommen und - was die Personenermittlungen angeht - auch ziemlich weit getrieben werden.

Aber auch bei den Zivilisten, bei denen die Anzeige etwa für Donald Rumsfeld auf geschäftliche Kontakte (also eine berufliche Verbindung) nach Deutschland verweist und von denen zumindest Rumsfeld und Gonzales auch aus beruflichen Gründen schon in Deutschland waren ${ }^{169}$, kann die Staatsanwaltschaft nicht ohne jede faktische Basis die von $\S 153 \mathrm{f}$ Abs. 1 StPO geforderte Prognose einfach behaupten. Auch hier müssten entsprechende faktische Hinweise für eine künftig nicht mehr zu erwartende Einreise erst im Rahmen aufzunehmender Ermittlungen gründlich geprüft werden, bevor dann gegebenenfalls für diejenigen unter den Angezeigten, für die solche Hinweise vorlägen, ein Verfahren nach $\S 153 f$ Abs. 1 StPO wieder eingestellt werden könnte.

Die Generalbundesanwaltschaft verkennt in ihrer gesamten Argumentation ein zentrales Konstruktionselement des $\S 153 \mathrm{f}$ Abs. 1 StPO: Wenn ihr keine positiven Hinweise auf eine tatsächlich bestehende Unwahrscheinlichkeit einer künftigen Einreise eines Verdächtigen vorliegen, muss sie ein Ermittlungsverfahren einleiten und dies weiterführen, bis sie entsprechende tatsächliche Anhaltspunkte hat ${ }^{170}$. Nichtwissen führt hier gerade nicht dazu, dass Ermittlungen unterlassen werden dürften, sondern verpflichtet vielmehr zu gerade diesen ${ }^{171}$. Dass sich dies weder in der Ausstattung der Generalbundesanwaltschaft für solche Fälle noch in ihrer Politik im Umgang mit Anzeigen nach dem VStGB niederschlägt, ändert nichts daran, dass dies die Gesetzeslage ist, an die sich die Staatsanwaltschaft schon nach Art. 1 Abs. 3, 20 GG zu halten hat.

Zusammenfassend lässt sich festhalten, dass die von der Generalbundesanwaltschaft herangezogene Norm des $§ 153 f$ Abs. 1 StPO hier keine Basis für eine Nichtaufnahme

167 Siehe die Strafanzeige von 2004 (Fn. 2), S.27, wo als Stationierungsorte für vier Angezeigte Heidelberg und Wiesbaden angegeben waren; vgl. auch KMR (Plöd) (Fn. 5), § 153f StPO, Rn.4.

168 LR (Beulke) (Fn. 110), § 153f StPO, Rn.16.

169 Siehe die Strafanzeige von 2006 (Fn. 6), S. 82 f.

170 Siehe auch KMR (Plöd) (Fn. 5), § 153f StPO, Rn.12.

171 In dieser Richtung auch LR (Beulke) (Fn. 110), § 153f StPO Rn.16. 
von Ermittlungen darstellt. Damit sind schon die Voraussetzung für eine Opportunitätsentscheidung nach $\S 153 \mathrm{f}$ Abs. 1 StPO hier nicht gegeben.

\section{Voraussetzungen einer Ermittlungsverweigerung nach $\S 153$ f Abs. 2 S. 2 StPO}

Eine Verfahrenseinstellung wäre damit nur unter den engeren Voraussetzungen des $\S 153 f$ Abs. 2 S. 2 StPO zulässig, also nur dann, wenn die Tat von einem internationalen oder einem vorrangig zuständigen anderen nationalen Gericht erfolgt und eine Auslieferung oder Überstellung an dieses Gericht zulässig und beabsichtigt ist ${ }^{172}$. Damit kann hier mangels Zuständigkeit eines internationalen Gerichts und der Möglichkeit irakischer Verfahren ${ }^{173}$ nur die US-Justiz gemeint sein ${ }^{174}$. Dies ist nach der zwar etwas komplizierten ${ }^{175}$, aber eindeutigen Systematik der Norm zwingende Voraussetzung dafür, dass ein Einstellungs- oder Nichtverfolgungsermessen überhaupt entsteht $^{176}$.

Dabei ist der Begriff der »Tat« im Kontext der StPO eindeutig auf eine konkrete Handlung konkreter Personen und nicht nur auf einen allgemeinen Sachverhaltskomplex zu beziehen ${ }^{177}$. Hierzu müssten konkrete Strafverfolgungsmaßnahmen seitens der USA erkennbar $\operatorname{sein}^{178}$. Im vorliegenden Verfahren versucht aber auch die Staatsanwaltschaft nicht mehr, ernsthaft eine Strafverfolgung der hier angezeigten Verantwortungsträger durch Behörden der USA zu behaupten. Die bisher dort abgehaltenen Militärverfahren gegen die unmittelbar auf den veröffentlichten Bildern zu sehenden Soldaten, in denen viel Wert auf die These gelegt wurde, es habe sich um Exzesstaten Einzelner gehandelt, in die keine Vorgesetzten involviert waren ${ }^{179}$, haben schon mehr als deutlich gemacht, dass die USA derzeit nicht gewillt sind, die Verantwortung militärischer und ziviler höherrangiger Verantwortungsträger zu untersuchen ${ }^{180}$. Durch den Erlass des heftig umstrittenen Military Commissions Act wurde die Berufung auf völkerrechtliche Normen

172 KMR (Plöd) (Fn. 5), § 153f StPO, Rn.12.

173 Dazu die Strafanzeige von 2006 (Fn. 6), S. 57 ff.

174 Basak (Fn. 5), S. 499 (509); Fischer-Lescano KJ 2005, S. 72 (75); Kurth ZIS 2006, S. 81 (84).

175 SK-StPO (Weßlau) (Fn. 114), § 153f StPO, Rn.4.

176 Singelnstein/Stolle ZIS 2006, S. 118 (119); siehe auch BR-DruckS. 29/02, S. 85 ff.

177 Dazu Ambos NStZ 2006, S. 434 (436 f.); Fischer-Lescano ILM 45 (2006), S. 115 (116); Keller GA 2006, S. 25 (35 f.); Kurth ZIS 2006, S. 81 (85); Meißner, Die Zusammenarbeit mit dem Internationalen Strafgerichtshof nach dem Römischen Statut (2003), S. 76; Singelnstein/Stolle ZIS 2006, S. 118 (121 f.).

178 Siehe KMR (Plöd) (Fn. 5), § 153f StPO, Rn.12; Bothe/Fischer-Lescano (Fn. 137), S. 11.

179 So z.B. die Sprachregelung in dem vom 21. Juli 2004 datierenden Bericht über Detaninee Operations Inspection von Generalleutnant Mikolashek, im Vorwort und auf S. ii (zugänglich unter <www.washingtonpost.com/wp-srv/world/iraq/abughraib/detaineereport.pdf >).

180 Ähnlich Ambos (Fn. 27), § 3 Rn.101; siehe auch die Strafanzeige von 2006 (Fn. 6), S. 16 f. Der kürzlich abgeschlossene Prozess gegen den Leiter der Verhörabteilung in Abu Ghraib im fraglichen Zeitraum Steve Jordan, der mit einer Rüge wegen Ungehorsams endete, war der einzige Prozess gegen einen Offizier wegen der dortigen Vorgänge. Gleichzeitig wird dieser Prozess auch in der offizielle Kommunikation des Pentagon als Schlusspunkt unter der Aufarbeitung dieser Vorgänge verstanden. Weitere Prozesse sind nicht geplant oder absehbar; siehe Pitzke (Fn. 16). 
und damit auch die Strafverfolgung nach dem Völkerstrafrecht für alle im Zuge des sogenannten »Kriegs « gegen den Terror begangenen Taten ausgeschlossen - eine gesetzliche Teilamnestie, die das klare Ziel hat, Verfahren gegen Verantwortungsträger auszuschließen ${ }^{181}$. Damit spricht im Moment absolut nichts dafür, dass die zivile und militärische Spitze der Armee, die diese Taten zu verantworten hat, in den USA einer Strafverfolgung ausgesetzt würde. Eine Berufung auf $\S 153 f$ Abs. 2 S. 2 StPO scheidet damit ebenfalls aus.

Damit ist aber festzuhalten, dass ein Einstellungs- oder Nichtverfolgungsermessen der Generalbundesanwaltschaft gar nicht eröffnet war, die Verweigerung der Aufnahme von Ermittlungen ist in diesem Falle nicht von $\S 153 \mathrm{f} \mathrm{StPO} \mathrm{gedeckt}{ }^{182}$. Für das anstehende Klageerzwingungsverfahren bedeutet dies, dass die Anzeigeerstatter zumindest mit dem aus prozessualen Gründen als Hilfsantrag ${ }^{183}$ formulierten Hauptanliegen, die Generalbundesanwaltschaft wenigstens zur Aufnahme förmlicher Ermittlungen zu zwingen, von Rechts wegen Erfolg haben müssten.

3. Ermessensausübung durch die Generalbundesanwaltschaft $-\S 153 \mathrm{f}$ Abs. $2 \mathrm{~S} .1 \mathrm{StPO}$

Dennoch seien einige Überlegungen zu der von der Generalbundesanwaltschaft nach zu Unrecht erfolgter - Annahme der Voraussetzungen des $\S 153 f$ Abs. 1 StPO getroffenen Ermessensentscheidung, keine Ermittlungen aufzunehmen, hier angestellt. Selbst wenn man $\S 153 \mathrm{f}$ Abs. 1 StPO mit der Staatsanwaltschaft unterstellen würde, bedeutet diese Norm ja nicht die Pflicht zur Verfahrenseinstellung beziehungsweise zur Nichtaufnahme von Ermittlungen ${ }^{184}$. Vielmehr wird auch dann nur ein Ermessen eröffnet, das die Staatsanwaltschaft pflichtgemäß, also orientiert an den gesetzlichen Vorgaben und willkürfrei, auszuüben hat ${ }^{185}$.

Für diese Ermessensausübung enthält zunächst $§ 153 f$ Abs. 2 S. 1 StPO Vorgaben. Nach dieser Norm »kann insbesondere« ein Verfahren eingestellt werden, wenn Opfer und Täter keine Deutsche waren, sich kein Tatverdächtiger im Inland aufhält und ein solcher Aufenthalt auch nicht zu erwarten ist und eine Strafverfolgung durch ein internationales oder ein anderes vorrangig zuständiges nationales Gericht (des Tatortstaates oder des Heimatstaates von Täter oder Opfer) die Tat verfolgt. Nach dem soeben Gesagten liegen diese Voraussetzungen, vor allem die anderweitige Strafverfolgung, aber nicht vor. Gerade deren Fehlen soll aber im Regelfall eine Verfahrenseinstellung und erst Recht eine Ermittlungsverweigerung ausschließen ${ }^{186}$.

181 In diesem Sinne auch die Kleine Anfrage aus der Fraktion von Bündnis 90/Die Grünen in BT-DruckS. 16/4099, S. 3; siehe auch die Strafanzeige von 2006 (Fn. 6), S. 27 ff., sowie Dorf JICJ 5 (2007, S. 10 (16 ff.); Stewart JICJ 5 (2007), S. 26 (36 f.).

182 Ambos NStZ 2006, S. 434 (437 f.); Basak (Fn. 5), S. 499 (512); Fischer-Lescano KJ 2005, S. 72 (85 f.); Singelnstein/Stolle ZIS 2006, S. 118 ff., insb. 120.

183 Klageerzwingungsantrag (Fn. 8), S. 3.

184 KMR (Plöd) (Fn. 5), § 153f StPO, Rn.12.

185 Siehe BVerfG NStZ 1982, S. 430.

186 LR (Beulke) (Fn. 110), § 153f StPO, Rn.42: »Wenn keine andere Strafverfolgung durch internationale oder nationale Jurisdiktionen gewährleistet ist, kann im Regelfall nicht eingestellt werden.« 
Damit stellt sich die Frage, wann denn außerhalb der in $\S 153 f$ Abs. 2 StPO genannten Fälle eine Verfahrenseinstellung oder eine Ermittlungsverweigerung zulässig sein kann. Zwar ist dies wegen der offenen Formulierung des $\S 153 \mathrm{f}$ Abs. 2 StPO grundsätzlich nicht ausgeschlossen. Allerdings müssen, wenn die Formulierung der Norm irgendeinen Sinn haben soll, die Anforderungen an die Gründe für eine Einstellung auBerhalb dieser Norm hoch $\operatorname{sein}^{187}$. Nur dies entspricht auch dem gesetzgeberischen Willen und dem Zweck der Norm, die Geltung des Legalitätsprinzips für Fälle des VStGB gegenüber sonstigen Auslandsfällen deutlich zu stärken. Dies entspricht auch den völkerrechtlichen Bestrafungspflichten aus den dem VStGB zugrundeliegenden Verträgen $^{188}$.

Der Gesetzgeber hat ausdrücklich Strafverfolgungsmaßnahmen auch dann nicht nur für möglich, sondern auch für geboten gehalten, wenn diese nur der Vorbereitung späterer Prozesse, auch im Ausland, dienen können ${ }^{189}$. Das von der Generalbundesanwaltschaft ins Feld geführte Argument, es sei kein erfolgreicher Verfahrensabschluss vor deutschen Gerichten zu erwarten ${ }^{190}$, kann also allein nicht eine Verfahrenseinstellung jenseits des $\S 153$ f Abs. 2 StPO tragen ${ }^{191}$. Für eine Effektuierung des Völkerstrafrechts ist angesichts des fehlenden Verfolgungswillens in den USA jeder Schritt eines offiziellen justiziellen Verfahrens im Ausland, also auch in Deutschland, essentiell wichtig, um die Täter auf internationaler Ebene klar zu delegitimieren ${ }^{192}$.

Demgegenüber hat die Staatsanwaltschaft die extreme Schwere der hier thematisierten Straftaten ebenso wenig in ihre Ermessensausübung einbezogen wie die Tatsache, dass hier der Sachverhalt weitestgehend vollständig bekannt und belegt ist ${ }^{193}$. Dies sind aber wesentliche Gesichtspunkte, die in eine Ermessensentscheidung hätten einbezogen werden müssen ${ }^{194}$.

Unhaltbar ist auch die Behauptung, es gäbe keine »erfolgversprechenden Ermittlungsansätze ${ }^{195}$. Einerseits enthält schon die Anzeige eine große Fülle diesbezüglicher Anregungen ${ }^{196}$, von der Vernehmung benannter Zeugen bis zu Rechtshilfeersu-

187 Nach KMR (Plöd) (Fn. 5), § 153f StPO, Rn.12 soll es gar bei Nichtvorliegen einer der genannten Voraussetzungen bei einer »Verfolgungspflicht im Inland « verbleiben. Siehe auch LR (Beulke) (Fn. 110), § 153f StPO, Rn.25.

188 Vgl. Bothe/ Fischer-Lescano (Fn. 137), S. 4 ff.; Schmidt, Externe Strafpflichten (2002), S. $131 \mathrm{ff}$.

189 BT-DruckS. 14/8892, S. 38; BT-DruckS. 16/4267, S. 2; LR (Beulke) (Fn. 110), §153f StPO, Rn.40; KK (Schoreit) (Fn. 76), § 153f StPO, Rn.9; Meyer-Goßner (Fn. 76), §153f StPO, Rn.8; Ambos NStZ 2006, S. 434 (435 f.); Basak (Fn. 5), S. 499 (508); Fischer-Lescano KJ 2005, S. 72 (86).

190 Verfügung der Generalbundesanwaltschaft vom 05.04.2007 (Fn. 7), S. 7.

191 Zappalà JICJ 4 (2006), S. 602 (608 ff.); Klageerzwingungsantrag (Fn. 8); unter 6.3.2.4; Kreß (Fn. 139), S. 11 f.

192 Siehe auch Zappalà JICJ 4 (2006), S. 602 (606).

193 So auch der Klageerzwingungsantrag (Fn. 8), unter 2.2. und 4.1.2.

194 Siehe etwa Beck'scher Online-Kommentar VwGO (Decker) (BeckOK-VwGO) (2007), $\S 114$ VwGO, Rn.21 ff.

195 Verfügung der Generalbundesanwaltschaft vom 05.04.2007 (Fn. 7), S. 7; dagegen der Klageerzwingungsantrag (Fn. 8), unter 4.1.2.3.

196 Strafanzeige von 2006 (Fn. 6), S. 91 ff. 
chen an die USA und andere Staaten. Wenn die Staatsanwaltschaft letztere per se als nicht erfolgversprechend verwirft ${ }^{197}$, überschreitet sie deutlich ihren Zuständigkeitsbereich. Denn hier sind sowohl bei der Bundesregierung wie seitens der USA politische Entscheidungen zu treffen, welche die Staatsanwaltschaft weder antizipieren kann noch diese vorwegnehmen dürfte ${ }^{198}$. Dass auch Personenermittlungen in den USA zumindest weiterführen können, zeigt etwa der Fall Khaled el-Masri, wo zwar nicht die Staatsanwaltschaft, sondern Journalisten die Klarnamen der an der Entführung beteiligten CIA-Agenten ermittelt haben, wo aber die Staatsanwaltschaft immerhin einen internationalen Haftbefehl erwirkt hat ${ }^{199}$. Zudem gibt es eine große Fülle veröffentlichter offizieller (Regierungs-) Dokumente aus den USA, die ausgewertet und in die Ermittlungen einbezogen werden könnten ${ }^{200}$. Auch liegen zumindest schriftlich Aussagen einer ganzen Reihe ehemaliger Gefangener sowohl aus Guantànamo ${ }^{201}$ als auch aus Abu Ghraib ${ }^{202}$ vor, die in ein Verfahren einbezogen werden könnten.

Andererseits stellt sich die Frage, ob nicht allein die über die Medien öffentlich zugänglich gemachten Beweismittel schon ausreichen, um hier einen hinreichenden Tatverdacht zu begründen und damit eine Anklageerhebung nach $\S 170$ Abs. 1 StPO zu rechtfertigen. Dann wären weitere Ermittlungsansätze hier in der Sache gar nicht nötig.

Zusammenfassend zeigt sich, dass die von der Generalbundesanwaltschaft genannten Gründe für eine Ermittlungsverweigerung außerhalb des $§ 153 f$ Abs. 2 StPO nicht tragfähig weil sowohl der Gesetzes- als auch der Faktenlage widersprechend sind.

\section{Die gerichtliche Überprüfbarkeit der Nichteröffnungsentscheidung}

Damit stellt sich die Frage, wie die nach dem bisher Gesagten falsche Entscheidung der Generalbundesanwaltschaft gerichtlich überprüft und korrigiert werden kann. Das OLG Stuttgart hatte im Klageerzwingungsverfahren bezüglich der ersten Anklage zunächst noch darauf verwiesen, dass nach $§ 172$ Abs. 2 S. 3 StPO eine Klageerzwingung ausgeschlossen $\operatorname{sei}^{203}$. Schon dies ist zweifelhaft, weil mit Recht darauf verwie-

197 Verfügung der Generalbundesanwaltschaft vom 05.04.2007 (Fn. 7), S. 7.

198 Vgl. auch Basak (Fn. 5), S. 499 (513) zur parallelen Frage der nicht von der Staatsanwaltschaft zu treffenden politischen Entscheidung zur Durchführung einer gerichtlich für zulässig erklärten Auslieferung. Zappalà JICJ 4 (2006), S. 602 (610) weist zu Recht auch darauf hin, dass dieses Kriterium im Falle eines Aufenthaltes eines ausländischen Verdächtigen nach $\S 153 \mathrm{f}$ Abs. 1 StPO auch keine Rolle spiele, obwohl in diesem Falle eine Kooperation der Heimatbehörden des Verdächtigen nicht weniger unwahrscheinlich sei.

199 Dazu etwa Gebauer unter <www.spiegel.de/politik/deutschland/0,1518,463453,00.html>.

200 Vgl. etwa Greenberg (Hrsg.), The Torture Papers (2005); siehe auch Hentoff (Fn. 19). Alvarez CWRJIL 37 (2006), S. 175 (176), spricht von einer »astonishing paper trail of apparent complicity in torture «. Die Veröffentlichung einer Vielzahl von Dokumenten hat die American Civil Liberties Union unter dem in den USA geltenden Freedom of Information Act erwirkt (unter <www.aclu.org/safefree/torture/torturefoia.html $>$ ).

201 Etwa unter <www.ccr-ny.org/v2/legal/september_11th/docs/Guantanamo_composite_state ment_FINAL.pdf $>$.

202 Siehe unter <www.washingtonpost.com/wp-srv/world/iraq/abughraib/swornstatements042 104.html>.

203 OLG Stuttgart NStZ 2006, S. 117 (118); KMR (Plöd) (Fn. 5), § 153f StPO, Rn.13. 
sen wird, dass der Gesetzgeber bei der Einordnung dieser Norm als $\S 153 f$ StPO die Aufzählung des $\S 173$ Abs. 2 S. 3 StPO nicht im Blick hatte, so dass hier durchaus ein Redaktionsversehen begründet werden könnte ${ }^{204}$.

Unabhängig davon ist aber anerkannt und auch vom OLG Stuttgart im Grundsatz akzeptiert worden, dass auch bei einer Einstellung aus Opportunitätsgründen eine Klageerzwingung dann möglich sein muss, wenn die Voraussetzungen für die im Rahmen der entsprechenden Norm zu treffenden Ermessensentscheidung entweder gar nicht vorlagen oder wenn die Ermessensausübung ihrerseits als willkürlich anzusehen ist ${ }^{205}$.

Unzutreffend hatte das OLG Stuttgart allerdings die Grenzen der Ermessensüberprüfung viel zu eng gesetzt ${ }^{206}$. Vor allem hat es die Unterscheidung zwischen den Voraussetzungen für eine Ermessensausübung und den bei der Ermessensentscheidung zu treffenden Erwägungen verkannt ${ }^{207}$. Gerade die Frage, ob nach $\S 153 f$ Abs. 1 StPO überhaupt ein Ermessen eröffnet ist, also ob sich eine verdächtige Person im Inland aufhält oder ein solcher Aufenthalt zu erwarten ist, ist in vollem Umfang einer gerichtlichen Überprüfung zugänglich ${ }^{208}$. Nach dem oben Gesagten müsste schon an dieser Stelle die Entscheidung der Generalbundesanwaltschaft verworfen werden, weil diese keine hinreichenden Gründe vorbringt, die gegen einen künftigen Aufenthalt gerade von Offizieren der US-Armee im Bundesgebiet sprechen. Ebenso wären bei Berufung auf $\S 153 f$ Abs. 2 S. 2 StPO die Merkmale des Satzes 1 Nr. 4, also die Frage einer anderweitigen Strafverfolgung, einer gerichtlichen Überprüfung zugänglich, weil gerade diese Voraussetzungen eine Einstellung erst ermöglicht ${ }^{209}$.

Im Rahmen einer Willkürprüfung bei Bejahung eines Einstellungsermessens wäre nach herkömmlichen Methoden zu prüfen, ob das Ermessen überhaupt erkannt und ausgeübt wurde und ob keine sachfremden Gründe die Entscheidung geprägt haben ${ }^{210}$. Letzteres wäre hier durchaus zu bedenken. Denn die Generalbundesanwaltschaft beruft sich nach dem oben Gesagten auf unplausible Tatsachenbehauptungen und agiert contra legem. Auch der Hinweis auf mangelnde Ressourcen der Generalbundesanwaltschaft für solche Fälle ${ }^{211}$ ist eine sachfremde Erwägung, weil dies höchstens zu einer Bedarfsanmeldung an das Bundesjustizministerium und zu einer entsprechenden Umorganisation innerhalb der Behörde führen müsste, nicht aber als Grund für eine Ermittlungsverweigerung dienen $\mathrm{kann}^{212}$. Eine auf einer solchen Basis ergehende Entscheidung kann nicht als willkürfrei angesehen werden.

204 Ambos NStZ 2006, S. 434 (437 f.); Klageerzwingungsantrag (Fn. 8), unter 4.1.2.1.

205 OLG Stuttgart NStZ 2006, S. 117 (118); Klageerzwingungsantrag (Fn. 8), S. unter 3.3; Ambos NStZ 2006, S. 434 (438).

206 Basak (Fn. 5), S. 499 (511 f.).

207 Hierzu etwa BeckOK-VwGO (Decker) (Fn. 194), § 114 VwGO, Rn.13.

208 Singelnstein/ Stolle ZIS 2006, S. 118 ff. (v.a. 120); Klageerzwingungsantrag (Fn. 8), unter 3.3.

209 Singelnstein/ Stolle ZIS 2006, S. 118 (120).

210 Siehe etwa BVerfG NStZ 1982, S. 430; vgl. auch § 114 VwGO sowie etwa BeckOKVwGO (Decker) (Fn. 194), § 114 VwGO, Rn.13 ff.

211 Verfügung der Generalbundesanwaltschaft vom 05.04.2007 (Fn. 7), S. 7.

212 Siehe auch Walther FS Heike Jung (2007), S. 1045 (1056); Klageerzwingungsantrag (Fn. 8), unter 2.2 (dort 2.). 
Nimmt man all dies ernst, müsste der Antrag auf gerichtliche Entscheidung nach $\S 172$ StPO erfolgreich für die Anzeigeerstatter verlaufen. Dass sich diese Erwartung als trügerisch erweisen kann, hat allerdings schon die Entscheidung des OLG Stuttgart zu der ersten Anzeige in dieser Sache gezeigt.

\section{Ausblick}

Wieder hat ein Gericht zu entscheiden, ob sich die in den 90er Jahren bezüglich der Barbareien im ehemaligen Jugoslawien noch sehr aktive deutsche Justiz ${ }^{213}$ entsprechend dem bei Erlass des VStGB deutlich gemachten gesetzgeberischen Willens auch für die Verfolgung schwerster Menschenrechtsverbrechen im Ausland zuständig fühlen muss, oder ob die bisherige Obstruktionshaltung der Generalbundesanwaltschaft weiter bestärkt wird. Es ist zu wünschen, dass das befasste Gericht die Verantwortung auch und gerade Deutschlands für eine erfolgreiche Implementierung hoher Standards in Menschenrechtsfragen und damit auch für eine Repression von schwersten Verbrechen gegen diese Menschenrechte ernst nimmt.

Der bisherige Umgang der Generalbundesanwaltschaft nicht nur mit diesem, sondern mit allen Fällen, die nach dem VStGB bei ihr angezeigt werden, lässt hier wenig Gutes erwarten. Wenn letztlich nur drei Personen jeweils in Teilzeit in der Behörde mit diesen Fällen befasst werden ${ }^{214}$, und deren Tätigkeit weitestgehend darin besteht, Bescheide zur Nichtaufnahme von Ermittlungen zu formulieren, lässt dies auf eine bewusst so gestaltete Politik der Behörde gegenüber der eigenen Verantwortung für die Verfolgung solcher Verbrechen schließen. Diese läuft darauf hinaus, das mit viel Verve erlassene VStGB einfach nicht anwenden zu wollen ${ }^{215}$. Damit wird dieses dem Gesetzgeber wichtige und prominent gewürdigte Projekt ${ }^{216}$ exekutiv ad absurdum geführt. Dies wäre ein Begräbnis Dritter Klasse für dieses wichtige Gesetz durch Anwendungsverweigerung ${ }^{217}$. Einer an Recht und Gesetz gebundenen Exekutive, also auch einer Staatsanwaltschaft, steht es aber nicht zu, unbequeme Gesetze einfach zu ignorieren.

Ob die Justiz diesem Versuch, die in Abu Ghraib und Guantànamo begangenen Verbrechen als das zu kennzeichnen, was sie waren und sind, nämlich von obersten staatlichen Stellen angeordnete Verbrechen gegen das Völkerrecht, doch noch zum Erfolg verhilft, ist schwer vorherzusehen. Es gibt fast keine gerichtliche Praxis zu diesen Fragen und die immensen politischen Implikationen eines solchen Falles sprechen eher dafür, dass wie im ersten Versuch auch insgesamt nicht ernsthaft vertretbare Begrün-

213 Hierzu etwa die Darstellung von Rissing-van Saan JICJ 3 (2005), S. 381 ff.

214 So die Antwort der Bundesregierung auf eine Kleine Anfrage aus der Fraktion Bündnis 90/ Die Grünen in BT-DruckS. 16/4267, S. 3.

215 Kaleck/ Wiener (Fn. 10), S. 23.

216 Siehe etwa Pressemitteilung 37/02 des Bundesministeriums der Justiz vom 27.06.2002.

217 Kritisch hierzu etwa amnesty international, Pressemitteilung vom 09.05.2006 zum Fall Almatov, unter <www2.amnesty.de/internet/deall.nsf/51a43250d61 caccfc1256aa1003d7d3 8/0366c5692eb3a3f0c12571690030f9a0?OpenDocument>. 
dungen herangezogen werden könnten, wenn nur das Ergebnis einer Abweisung des Antrags erzielt werden können.

Die Rechtswissenschaft kann hier nicht mehr tun, als solche Begründungen zu analysieren und ihre Fehlerhaftigkeit aufzuzeigen. Übrig bleibt dann ein politisch getriebener Dezisionismus, dem die rechtliche Legitimation fehlt. Diesem Ziel dient der vorliegende Text, und dem muss auch die weitere Begleitung aller anhängig gemachten Verfahren nach dem VStGB in der Hoffnung dienen, dass sich irgendwann Recht und Gesetz gegen extern motivierte Widerstände durchsetzen können. 